\title{
Practical Considerations with Radar Data Height and Great Circle Distance Determination
}

\author{
MARK A. AsKelson, ${ }^{a}$ Chris J. Theisen, ${ }^{\mathrm{b}}$ AND RANDAll S. JOHNSON ${ }^{\mathrm{a}}$ \\ ${ }^{a}$ Department of Atmospheric Sciences, University of North Dakota, Grand Forks, North Dakota \\ ${ }^{\mathrm{b}}$ Northern Plains Unmanned Aircraft Systems Test Site, Grand Forks, North Dakota
}

(Manuscript received 6 June 2019, in final form 30 October 2020)

\begin{abstract}
Owing to their ease of use, "simplified" propagation models, like the equivalent Earth model, are commonly employed to determine radar data locations. With the assumption that electromagnetic rays follow paths of constant curvature, which is a fundamental assumption in the equivalent Earth model, propagation equations that do not depend upon the spatial transformation that is utilized in the equivalent Earth model are derived. This set of equations provides the true constant curvature solution and is less complicated, conceptually, as it does not depend upon a spatial transformation. Moreover, with the assumption of constant curvature, the relations derived herein arise naturally from ray tracing relations. Tests show that this new set of equations is more accurate than the equivalent Earth equations for a "typical" propagation environment in which the index of refraction $n$ decreases linearly at the rate $d n / d h=-1 / 4 a$, where $h$ is height above ground and $a$ is Earth's radius. Moreover, this new set of equations performs better than the equivalent Earth equations for an exponential reference atmosphere, which provides a very accurate representation of the average atmospheric $n$ structure in the United States. However, with this $n$ profile the equations derived herein, the equivalent Earth equations, and the relation associated with a flat Earth constant curvature model produce relatively large height errors at low elevations and large ranges. Taylor series approximations of the new equations are examined. While a second-order Taylor series approximation for height performs well under "typical" propagation conditions, a convenient Taylor series approximation for great circle distance was not obtained.
\end{abstract}

KEYWORDS: Measurements; Radars/Radar observations; Remote sensing

\section{Introduction}

Analysis of radar data often requires knowledge of their locations within the atmosphere. Numerous techniques for estimating radar data locations exist, including, in order of decreasing complexity, direct solution methods [the direct solution of Maxwell's equations, including the method of moments (Harrington 1993) and the finite-difference time-domain (FDTD) technique (Taflove and Hagness 2005)]; methods based on approximating solutions to the two-dimensional wave equation, which is a solution to Maxwell's equations (including mode theory and parabolic equation techniques; Levy 2000); ray tracing, which is a method for determining the paths of electromagnetic waves by using geometry and successive applications of Snell's law of refraction and the law of reflection (Born and Wolf 1999); and "simplified" propagation models, which are defined herein to be those that utilize assumptions regarding the spatial structure of the index of refraction $n$ of the atmosphere to enable the computation of electromagnetic ray paths without the need for a more sophisticated technique such as ray tracing. The best known of the simplified propagation models is the equivalent Earth model, which was introduced by Schelleng et al. (1933).

Despite the availability of sophisticated techniques for determining characteristics (location, intensity, etc.) of radar beams, analysts commonly use techniques such as the equivalent Earth model owing to their relative simplicity and utility in many situations. However, radar beam propagation can vary appreciably from the solutions obtained using simple propagation

Corresponding author: Mark A. Askelson, askelson@aero.und.edu models, including nonsimple propagation phenomena such as ducting and reflections, when conditions vary significantly from the "typical" conditions assumed for the equivalent Earth model. ${ }^{1}$ Interestingly, a proposed solution in this situation is the application of the equivalent Earth model in layers to better estimate the impacts of atypical conditions (Gao et al. 2006).

Herein, the purpose is to

1) present a new simplified propagation model that depends upon the constant-curvature assumption that forms the basis of the equivalent Earth approximation but that does not require the slightly imperfect spatial transformation that is utilized in the equivalent Earth model to produce a straight ray, and

2) provide practical guidance regarding errors associated with commonly used simplified propagation models as a function of elevation and range for ground-based radars and tropospheric phenomena.

It is shown that for typical applications the equations associated with the new simplified propagation model are as easy to use as those from the equivalent Earth model. However, for a reference atmosphere in which $d n / d h=-1 / 4 a$, where $n$ is the index of refraction, $h$ is above radar-ground-level height, and $a$ is Earth's radius, the new simplified propagation model

\footnotetext{
${ }^{1}$ It is noted that the meaning of "significant variation from typical conditions" depends upon the application as that determines the acceptable level of accuracy. Herein, this concept is better defined for "typical" (tropospheric) weather radar applications.
} 
provides more accurate great circle distance values while providing essentially equivalent height values. Moreover, the new simplified propagation model is conceptually simpler in that it does not require the imperfect spatial transformation that is utilized in the equivalent Earth model.

The need for guidance regarding errors stems from the lack of such information in the published literature. Information regarding errors associated with simplified propagation equations is oftentimes provided in a general sense (e.g., less than some value) using approximate equations such as in Schelleng et al. (1933), is not plotted directly but must be estimated from plots of heights or great circle distance (e.g., Doviak and Zrnić 1993 , p. 23), or is presented in terms of other variables, such as total bending, that are not as useful to meteorologists as are height and great circle distance errors (e.g., Bean and Dutton 1966, section 3.8). Thus, there is a need for direct plots of height and great circle distance errors associated with simplified propagation models that can be easily used to understand error magnitudes and potential impacts as a function of radar elevation and range. This is underscored by the results of an informal poll of analysts who developed commonly used meteorological software in which radar heights and great circle distances are used to transform radar data locations from radar-centric to Earth-centric coordinates. It was found that simplified propagation models were commonly used in these software packages, but that most analysts were uncertain of error magnitudes and the spatial structure of errors (as a function of elevation and range) associated with the use of simplified propagation models.

The layout of this manuscript is as follows. The new simplified propagation equations are presented in section 2 . Practical guidance regarding errors associated with commonly used simplified propagation models, including the new equations, the equivalent Earth equations, the flat Earth approximation equations, and those associated with the newly developed relations for a flat Earth with refraction (Davies-Jones et al. 2019), is provided in section 3. A discussion is provided in section 4 , which is then followed by conclusions in section 5 .

\section{Propagation equations}

\section{a. Basic assumptions}

For all propagation models considered herein, it is assumed that $n$ varies only in the vertical. Thus, temperature, pressure, and humidity are assumed to be horizontally homogeneous (the atmosphere is assumed to be spherically stratified for a spherical Earth) resulting in propagation in a two-dimensional plane. Moreover, $n$ is assumed to be smoothly changing [i.e., within one wavelength of an electromagnetic wave the relative changes in refractivity $N$, where $N=(n-1) \times 10^{6}$, are small] so that ray theory applies.

\section{b. FENR model}

The simplest equations result when Earth is assumed to be flat and the atmosphere is assumed to be nonrefractive ( $n$ constant). For this flat Earth, no refraction (FENR) model,

$$
h=h_{a}+r \sin \theta
$$

and

$$
s=r \cos \theta,
$$

where $h$ is the above radar-ground-level height, $s$ is the great circle distance [at mean sea level (MSL)], $h_{a}$ is above radarground-level antenna height, $r$ is range, and $\theta$ is the initial elevation angle of a ray (see Fig. 1).

\section{c. Constant ray curvature solutions}

The purpose is to determine, assuming that rays have constant curvature, the above radar-ground-level height $h$ and great circle distance $s$ (at MSL; see Fig. 1) of a radar datum for radars for which the elevation $\theta=0^{\circ}$ is in a direction that is perpendicular to the line emanating from the center of Earth through the radar. This problem has been considered in detail by previous investigators (e.g., Doviak and Zrnić 1993, 14-28) and depends upon rays following constant curvature paths, which is discussed subsequently.

\section{1) RAY CURVATURE}

Given the commonly applied assumptions of a spherically stratified atmosphere and relatively smoothly varying $n$ fields, the following of constant-curvature paths depends primarily upon the assumptions that $h+h_{R_{-} \mathrm{MSL}} \ll a$, where $h_{R_{-} \mathrm{MSL}}$ is the MSL elevation at the radar location, and that $n$ is linearly dependent upon height. The general equation for ray curvature $C$ in polar coordinates (Doviak and Zrnić 1993, p. 19) can be expressed, by using the fact that the differential of the angle at $O$ in Fig. 1 is given by $d s / a$, as

$C=\frac{\left[\left(a+h_{R_{-} \mathrm{MSL}}+h\right)^{2}+2 a^{2}\left(\frac{d h}{d s}\right)^{2}-\left(a+h_{R_{-} \mathrm{MSL}}+h\right) a^{2} \frac{d^{2} h}{d s^{2}}\right]}{\left[\left(a+h_{R_{-} \mathrm{MSL}}+h\right)^{2}+a^{2}\left(\frac{d h}{d s}\right)^{2}\right]^{3 / 2}}$.

By

1) substituting for $d^{2} h / d s^{2}$ using

$$
\begin{aligned}
& \frac{d^{2} h}{d s^{2}}-\left[\frac{2}{\left(a+h_{R_{-} \mathrm{MSL}}+h\right)}+\frac{1}{n} \frac{d n}{d h}\right]\left(\frac{d h}{d s}\right)^{2} \\
& -\left(\frac{a+h_{R_{-} \mathrm{MSL}}+h}{a}\right)^{2}\left(\frac{1}{a+h_{R_{-} \mathrm{MSL}}+h}+\frac{1}{n} \frac{d n}{d h}\right)=0,
\end{aligned}
$$

which is a general equation for a ray propagating in a spherically stratified atmosphere (Appleton 1946; Hartree et al. 1946), and

2) substituting for $d h / d s$ using

$$
\frac{d h}{d s}=\left[\frac{\left(a+h_{R \_\mathrm{MSL}}+h\right)^{4} n^{2}-\left(a+h_{R_{-} \mathrm{MSL}}+h\right)^{2} C_{\mathrm{SL}}^{2}}{a^{2} C_{\mathrm{SL}}^{2}}\right]^{1 / 2},
$$

which arises by relating $d h$ to $d s$ in a spherically stratified atmosphere (Doviak and Zrnić 1993, appendix A) 


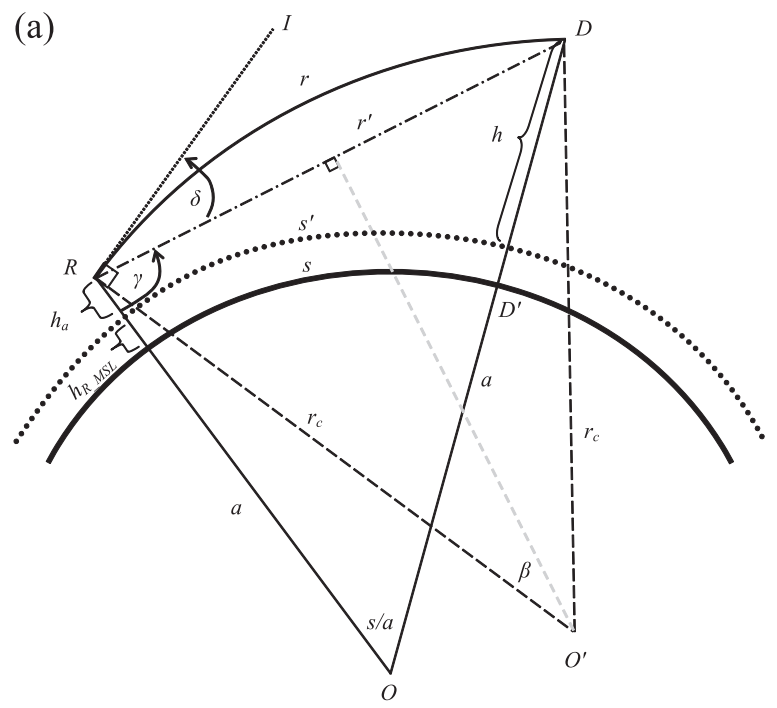

(b)

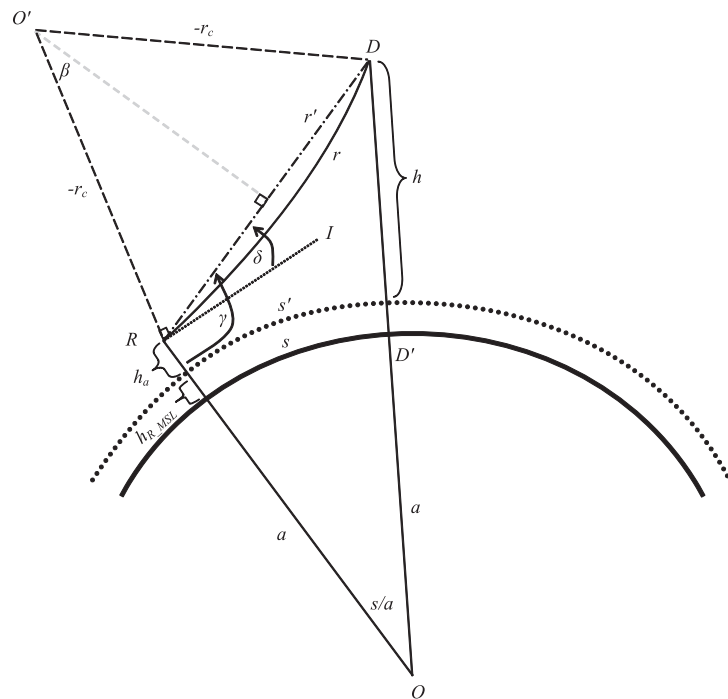

(c)

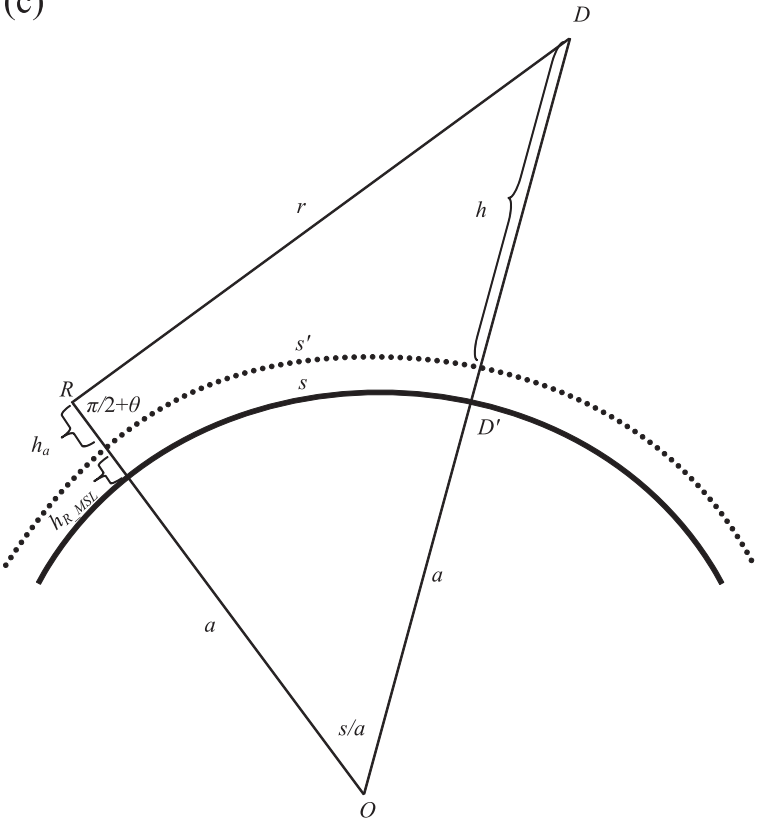

FIG. 1. Illustration of the above radar-ground-level height $h$ and great circle distance (at mean sea level) $s$ determination problem for a ray propagating in an atmosphere in which (a) $n$ decreases linearly with height, (b) $n$ increases linearly with height, and (c) $n$ is constant. After application of the approximations discussed in the paragraph following that containing (6), the ray in (a) follows a path of constant curvature with a radius of curvature $r_{c}$, the ray in (b) follows a path of constant curvature with a radius of curvature $-r_{c}$, and the ray in (c) follows a straight line. $R$ denotes the radar location, $h_{a}$ is the (radar) antenna height, $h_{R_{-} \mathrm{MSL}}$ is the MSL elevation at the radar location, $r$ is the true (not apparent) slant range, $D$ denotes the datum location, $r^{\prime}$ is the length of the chord RD [equal to $r$ in (c)], RI denotes the ray path in a homogeneous (nonrefractive) atmosphere [denoted by RD in (c)], $\gamma$ is the angle between the lines OR and RD, $\delta$ is the angle between the lines RD and RI, $\theta$ is initial elevation angle, $D^{\prime}$ denotes the projection of the datum location to mean sea level, $s^{\prime}=\left[\left(a+h_{R_{-} \mathrm{MSL}}\right) / a\right] s$ corresponds to $s$ but is at the elevation of $h_{R_{-} \mathrm{MSL}}, a$ is the radius of Earth, $O$ denotes the center of Earth, $O^{\prime}$ denotes the center of the circle that contains the arc RD, and $\beta$ is half of the angle between $O^{\prime} R$ and $O^{\prime} D$. For illustration purposes, features like antenna height $h_{a}$ and the relative curvatures of Earth's surface and of the arc RD are not drawn to scale.

and wherein $C_{\mathrm{SL}}$ arises from Snell's law of refraction and is given by $C_{\mathrm{SL}}=\left(a+h_{R_{-} \mathrm{MSL}}+h_{0}\right) n\left(h_{0}\right) \cos \left[\theta\left(h_{0}\right)\right]$ with subscript zeroes indicating values at a reference location, one obtains

$$
C=\frac{1}{r_{c}}=-\frac{d n}{d h} \frac{n\left(h_{0}\right)}{n^{2}(h)}\left[\frac{1+\frac{h_{R_{-} \mathrm{MSL}}+h_{0}}{a}}{1+\frac{h_{R_{-} \mathrm{MSL}}+h}{a}}\right] \cos \left[\theta\left(h_{0}\right)\right],
$$

where $r_{c}$ is the radius of curvature. It is noted that this relation can also be derived from the equation for the local curvature of a ray, such as Eq. (5) in Millington (1957), by utilizing Snell's law for spherically stratified media $\left(a+h_{R \_\mathrm{MSL}}+h_{0}\right) n\left(h_{0}\right) \cos \left[\theta\left(h_{0}\right)\right]=\left(a+h_{R \_\mathrm{MSL}}+h\right)$

$n(h) \cos [\theta(h)]$ (also known as Bouger's, or Bouguer's, law) (Bean and Dutton 1966, section 3.1; Kursinski et al. 2000; Coleman 2004).

If $h+h_{R_{-} \mathrm{MSL}} \ll a$ (herein the focus is the troposphere for which $h+h_{R \_ \text {MSL }} \leq 20 \mathrm{~km}$ ) and $n$ varies linearly with height, ray curvature varies with height owing only to the variation of $n^{2}$ with height. Because $n(h)$ can be expressed as $1+\varepsilon(h)$, where $\varepsilon(h)$ is on the order of $10^{-4}$, and $n^{2}(h)=1+2 \varepsilon(h)+$ $\varepsilon^{2}(h) \approx 1$, the curvature of a radar ray is approximately constant if $n$ varies linearly with height.

It is noted that the curvature of a radar ray is approximately constant if $n$ varies linearly with height regardless of the initial elevation angle of the beam. However, the value of the curvature does vary with initial beam elevation. This may be 
confusing since with techniques such as the equivalent Earth model a constant curvature value is commonly used. This can be reconciled by considering that as $\theta\left(h_{0}\right)$ increases the curvature decreases and, thus, the radius of curvature increases. Thus, if one starts with a relatively large radius of curvature wherein curvature effects are relatively slight and moves to higher elevations at which curvature effects are even weaker, the impact of the change of curvature is relatively small. Moreover, at high elevations radar rays reach altitudes of $\sim 20 \mathrm{~km}$ rapidly, further decreasing the impacts of nonzero curvature. Even so, it can be helpful to allow ray curvature to vary with $\cos \left[\theta\left(h_{0}\right)\right]$ (Davies-Jones et al. 2019). Herein, ray curvature is generally assumed to not vary with initial beam elevation.

\section{2) SEECC MODEL}

A common approach to determining $h$ and $s$ is the use of the spherical equivalent Earth constant curvature (SEECC) model, which is composed of two components (Schelleng et al. 1933; Bean and Dutton 1966, 56-59; Doviak and Zrnić 1993, 18-21). The first is the assumption that rays follow constantcurvature paths. The second is a transformation from the actual spatial configuration to one in which the ray path is straight, implying a "bending" of both the ray path and Earth's surface (cf. Fig. 1). In this spatial transformation the radius of a fictitious "equivalent" Earth is determined so that $h$ and $s$ are essentially unchanged. With this model, Doviak and Zrnić (1993) obtain the relations

$$
\begin{aligned}
h= & {\left[r^{2}+\left(k_{e} a+h_{R_{-} \mathrm{MSL}}+h_{a}\right)^{2}\right.} \\
& \left.+2 r\left(k_{e} a+h_{R_{-} \mathrm{MSL}}+h_{a}\right) \sin \theta\right]^{1 / 2}-\left(k_{e} a+h_{R_{-} \mathrm{MSL}}\right)
\end{aligned}
$$

and

$$
s=\left(k_{e} a\right) \sin ^{-1}\left[\frac{r \cos \theta}{\left(k_{e} a+h_{R_{-} \mathrm{MSL}}\right)+h}\right],
$$

where $k_{e}$ is the constant in the equivalent Earth model (usually it is assumed that $k_{e}=4 / 3$ ) and the situation where $h_{R_{-} \mathrm{MSL}}$ and $h_{a}$ are nonzero has been incorporated.

A related equation is

$$
h=h_{a}+r \sin \theta+\frac{\cos ^{2} \theta}{2\left(k_{e} a+h_{R_{-} \mathrm{MSL}}+h_{a}\right)} r^{2},
$$

which is a slight modification ( $h_{a}$ and $h_{R_{-} \text {MSL }}$ have been incorporated) of an approximation obtained by Bent et al. (1950). To obtain (9), Bent et al. (1950) utilized the SEECC model and made several simplifying assumptions. Since (9) is equivalent to the second-order Taylor series approximation of (7) obtained by expanding about $r / a=0$, Bent et al. (1950) effectively illustrate what is neglected in that Taylor series approximation.

\section{3) Flat EARTh CONSTANT CURVATURE MODEL}

Davies-Jones et al. (2019) developed a general means for producing relations for $h$ and $s$ given the assumption of constant ray curvature. This approach can produce relations for the case of spatial transformation to a flat Earth. The latter set of relations support simulation of radar signatures and are given by

$$
h=h_{a}+r_{c f}\left\{\sin \left(\frac{r}{r_{c f}}\right) \sin \theta-\left[1-\cos \left(\frac{r}{r_{c f}}\right)\right] \cos \theta\right\}
$$

and

$$
s=r_{c f}\left\{\sin \left(r / r_{c f}\right) \cos \theta+\left[1-\cos \left(r / r_{c f}\right)\right] \sin \theta\right\},
$$

where $r_{c f}=1 /\left\{\left[\left(1 / r_{c} \cos \theta\right)-(1 / a)\right] \cos \theta\right\}$ is the radius of curvature corresponding to a flat Earth. ${ }^{2}$

4) SPHERICAL EARTH CONSTANT CURVATURE MODEL

\section{(i) Derivation}

While the SEECC and flat Earth constant curvature (FECC) models provide very useful relations, it turns out that the second step in developing those models-the spatial transformation -is not required for developing useful propagation equations. All that is required is the assumption that rays follow constant curvature paths.

The problem for downward bending rays $\left(r_{c}>0\right)$ is illustrated in Fig. 1a. The assumption that radar rays follow paths of constant curvature implies that the curved ray path from $R$ to $D$ (from the radar location to the datum location) is an arc of a circle of radius $r_{c}$, the value of which is given by (6). With knowledge of $r_{c}, \gamma$, and $r^{\prime}$, the laws of cosines and sines can be used to develop relations for $h$ and $s$. The value of $r$ ' is obtained by noting, from the circle sector $O^{\prime} R D$, that $\beta=r / 2 r_{c}$. With this,

$$
r^{\prime}=2 r_{c} \sin \left(\frac{r}{2 r_{c}}\right)
$$

The value of $\gamma$ is determined by noting that the angle between $O R$ and $R I$ is $\pi / 2+\theta$ and, thus, that $\gamma=\pi / 2+\theta-\delta$. From the triangle with vertices at $O^{\prime}, R$, and the midpoint of the line RD, and the fact that $O^{\prime} R$ and $R I$ are perpendicular, $\delta=\beta=r / 2 r_{c}$.

Before presenting the equations for a downward bending ray, the situations in which rays bend upward $\left(r_{c}<0\right)$ is considered (Fig. 1b). Because the geometry for this situation is different, one might expect the resulting equations to be different. As with the $r_{c}>0$ case, needed relations are derived once $r_{c}, r^{\prime}$, and $\gamma$ are known. From the circle sector $O^{\prime} R D$, $\beta=r / 2\left(-r_{c}\right)$ and, thus, $r^{\prime}=2\left(-r_{c}\right) \sin \left[r / 2\left(-r_{c}\right)\right]=2 r_{c} \sin \left(r / 2 r_{c}\right)$. Consequently (12) holds in this situation as well. To determine $\gamma$ it is noted that the angle between $O R$ and $R I$ is $\pi / 2+\theta$ and, thus, that $\gamma=\pi / 2+\theta+\delta$. From the triangle with vertices at $O^{\prime}, R$, and the midpoint of the line $R D$, and the fact that $O^{\prime} R$ and $R I$ are perpendicular, $\delta=\beta=r / 2\left(-r_{c}\right)$. Thus, $\gamma=\pi / 2+$ $\theta-r / 2 r_{c}$. This is the same result as that obtained for the $r_{c}>0$ case. Thus, the set of equations for this situation is the same as

\footnotetext{
${ }^{2}$ The $\cos \theta$ terms in the $r_{c f}$ relation can be set to 1 if one ignores the dependence of ray curvature on elevation angle and uses a constant $r_{c}$ value.
} 
those for the $r_{c}>0$ case. The difference, of course, is that the value of $r_{c}$ is negative in this situation.

The other geometrical configuration is a straight ray (Fig. 1c), which occurs when the atmosphere is homogeneous (nonrefractive). In that case, relations are developed through straightforward applications of the laws of cosines and sines. It is noted that this model, which is a subset of the spherical Earth constant curvature (SECC) model, is termed the spherical Earth no refraction (SENR) model.

A constant curvature model will not necessarily produce correct results when $\theta= \pm 90.0^{\circ}$. When $\theta= \pm 90.0^{\circ}$, the assumed spherically stratified atmospheric structure results in no ray refraction. In this case, the geometry illustrated in Figs. 1a and $1 \mathrm{~b}$ break down and the radar ray travels in a straight line. In that situation, when $\theta=+90.0^{\circ}, h=h_{a}+r$, and when $\theta=-90.0^{\circ}, h=h_{a}-r$. This condition is labeled the no refraction condition (NRC). It is noted that (1)-(2) and (7)-(9) satisfy the NRC, while (10)-(11) satisfy the NRC in the limit if $r_{c f}$ varies with $\cos \theta\left(r_{c f}\right.$ is infinite at $\left.\theta= \pm 90.0^{\circ}\right)$.

The equation set is presented in Table 1 , which provides a compact format for presenting the equations, their origin, and relevant comments. This table also provides Taylor series approximations, which Gao et al. (2006) show may be useful.

Some relevant properties of this equation set are as follows:

1) Equations (13c) and (14d) are recommended for use in typical refractive conditions in which $r_{c}=4 a$ (corresponds to $k_{e}=4 / 3$ in the SEECC model) owing to their accuracy (illustrated below) and their satisfaction of the NRC.

2) Equations $[15 \mathrm{a}(2)]$ is a reexpression of $[15 \mathrm{a}(1)]$ and is presented for completeness since some applications (e.g., computation of vertical derivatives) may require the computation of $r$ given $s$ and $\theta$.

3) The accuracy of approximate forms and Taylor series generally depend upon $r_{c}$ being large. With increasing superrefraction, the performance of these equations degrades.

4) A comparison of (13)-(15) with (16)-(19) shows that the $r^{\prime}$ and $r / 2 r_{c}$ terms in (13)-(15) incorporate corrections owing to index of refraction variations.

5) The approximation (14d) does not contain any refractioncorrection terms beyond those that may be present in the $h$ relation used therein. While this approximation performs well without any of these correction terms, ignoring these terms in (14a) [i.e., approximating $r^{\prime} \cos \left(\theta-r / 2 r_{c}\right)$ as $r \cos \theta$ ] does not result in as accurate of an approximation. Differences between (14a) with refraction-correction terms ignored [with $h$ from (13a)] and (14a) [with $h$ from (13a)] for an altitude of $20 \mathrm{~km}$ [from (13c)], $r_{c}=4 a(a=$ $6370 \mathrm{~km})$, and $h_{R \_\mathrm{MSL}}=h_{a}=0 \mathrm{~km}$ range from $+51.01 \mathrm{~m}$ at $0.0^{\circ}$ elevation to $-79.34 \mathrm{~m}$ at $2.5^{\circ}$ elevation to $-9.54 \mathrm{~m}$ at $55.0^{\circ}$ elevation to $-7.82 \mathrm{~m}$ at $90.0^{\circ}$ elevation.

6) Comparison of (1) and (2), (20) and (21), and (22) and (23) shows that the SECC Taylor series approximations provide a convenient separation of terms that correspond to the FENR approximation and that result from Earth curvature (EC) and ray curvature (RC). The corresponding Taylor series approximation for $h$ in the equivalent Earth model
(9) provides separation of the FENR approximation, but does not provide separation of EC and RC terms.

7) To second order (20a)-(20c) are equivalent and satisfy the NRC.

8) The correction term $a /\left(a+h_{R_{-} \mathrm{MSL}}+h_{a}\right)$ that is present in (21) and (23) is associated with projecting downward from $h_{R_{-} \mathrm{MSL}}+h_{a}$ to mean sea level.

9) Equations (16) and (17) have forms that are similar to (7) and (8), with $a$ in (16) and (17) taking the place of $k_{e} a$ in (7) and (8). This similarity in form is expected as in both situations the ray path is a straight line over a curved Earth surface.

\section{(ii) Relation to ray tracing relations}

As shown in appendix B, with $r_{c}$ constant, a relation for $d h / d \ln n$ can be derived from (6). Substitution of this into (A4) and simplification of terms in the resulting radicand produce a result that is the derivative of the solution to a quadratic equation. That quadratic equation, when expressed in terms of $h$, is

$$
\begin{aligned}
h \approx & {\left[r^{2}+\left(a+h_{R_{-} \mathrm{MSL}}+h_{a}\right)^{2}+2 r\left(a+h_{R_{-} \mathrm{MSL}}+h_{a}\right)\right.} \\
& \left.\times\left(\sin \theta-\frac{r}{2 r_{c}} \cos \theta\right)\right]^{1 / 2}-\left(a+h_{R_{-} \mathrm{MSL}}\right) .
\end{aligned}
$$

This result is (13c). The SEECC equation for $h(7)$ arises from (13) if $r_{c} \rightarrow \infty$ and $a$ is replaced with $k_{e} a$. Thus, with the assumption of constant $r_{c}$ the SECC model derived herein arises naturally from the ray tracing relations whereas the SEECC model requires the additional transformation that is inherent to that model.

\section{Results}

Because the focus is on tropospheric applications, results are computed up to altitudes of $20 \mathrm{~km}$ MSL. Unless noted otherwise, $h_{R_{-} \mathrm{MSL}}=h_{a}=0 \mathrm{~km}$ and $a=6370 \mathrm{~km}$. These are chosen as reference values since a range of values is possible. The relative dependence upon $h_{R_{-} \mathrm{MSL}}, h_{a}$, and $a$ is relatively weak. For instance, at $20 \mathrm{~km} \mathrm{MSL}$ and with $\theta=0^{\circ}$, a radar located $50 \mathrm{~m}$ above the peak of Mount Everest ( $8.848 \mathrm{~km}$ elevation) has a difference between (7) and (13a) that is $3 \mathrm{~m}$ larger than that for a radar with $h_{R_{-} \mathrm{MSL}}=h_{a}=0 \mathrm{~km}$. Similarly, at $20 \mathrm{~km}$ MSL with $\theta=0^{\circ}$ and $h_{R_{-} \mathrm{MSL}}=h_{a}=0 \mathrm{~km}$, using $a=$ $6356.752 \mathrm{~km}$ or $a=6378.137 \mathrm{~km}$ results in altitude differences between (7) and (13a) that are within $1 \mathrm{~m}$ of the difference obtained with $a=6370 \mathrm{~km}^{3}$

\section{a. "Typical" refractive conditions}

The gradient of $n$ in the lowest portion of the troposphere, where most of the bending of rays occurs, has a typical value of $d n / d h=-1 / 4 a$ (Doviak and Zrnić 1993, section 2.2.3). If one

\footnotetext{
${ }^{3}$ The $a=6356.752 \mathrm{~km}$ and $a=6378.137 \mathrm{~km}$ values are the minor and major axes of the WGS84 reference ellipsoid (NIMA 2000).
} 


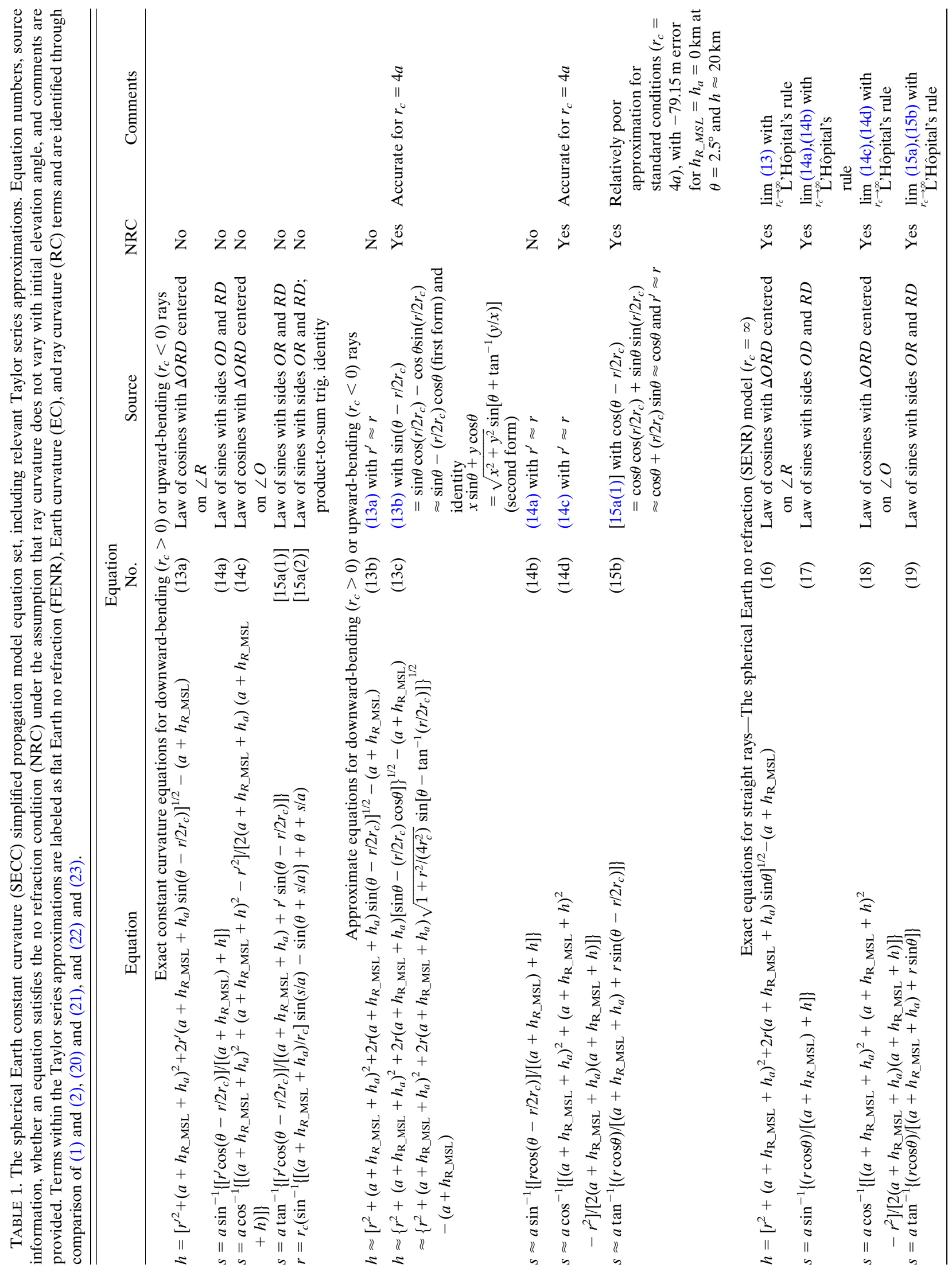




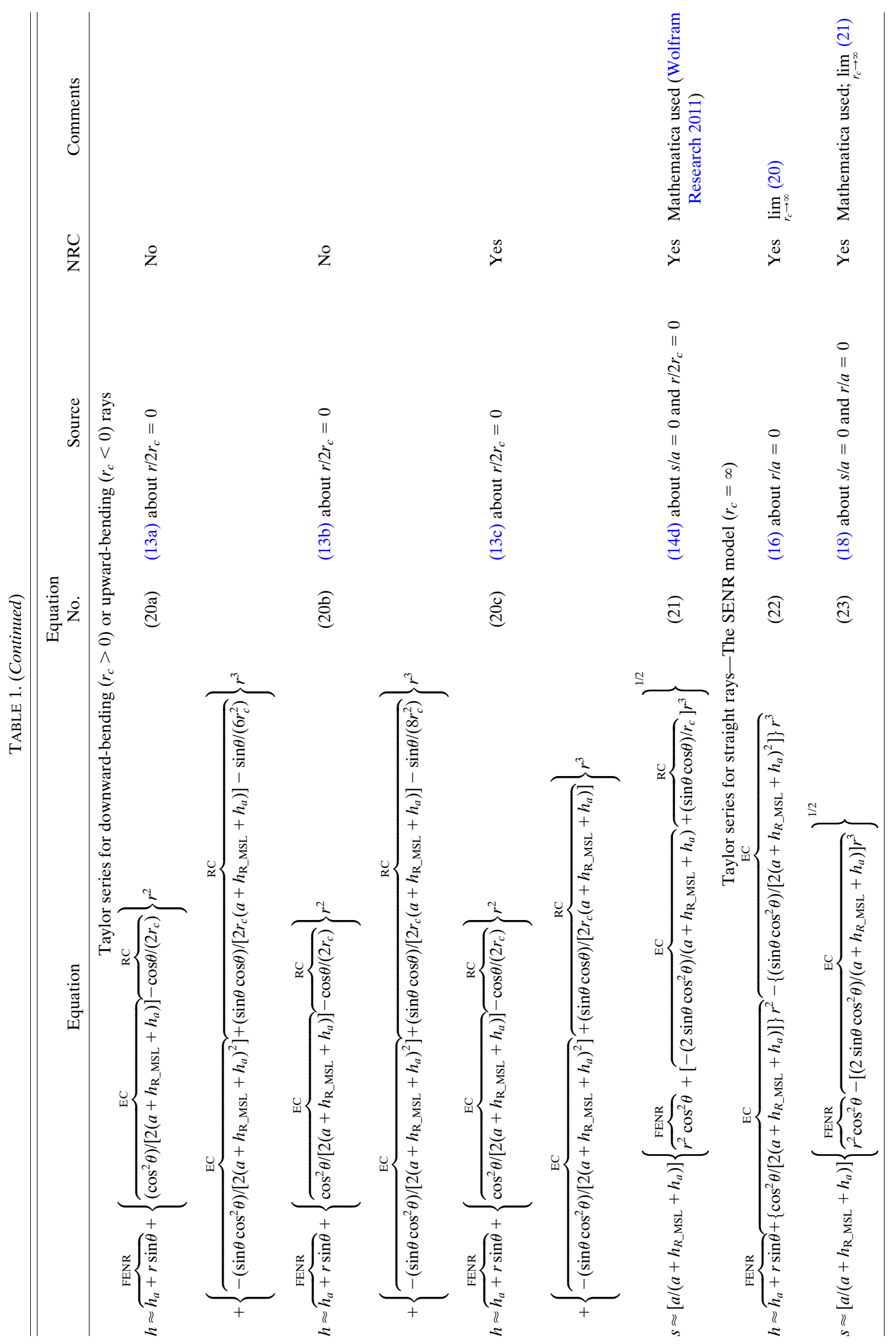




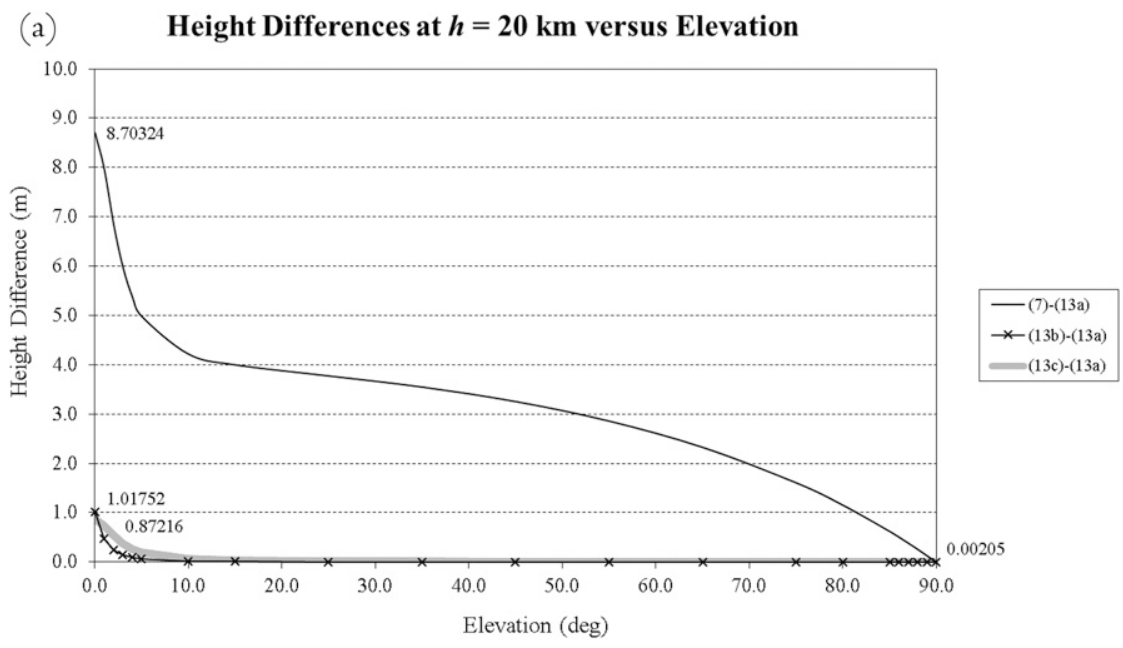

(b) Great-Circle Distance Differences at $\boldsymbol{h}=\mathbf{2 0} \mathrm{km}$ versus Elevation

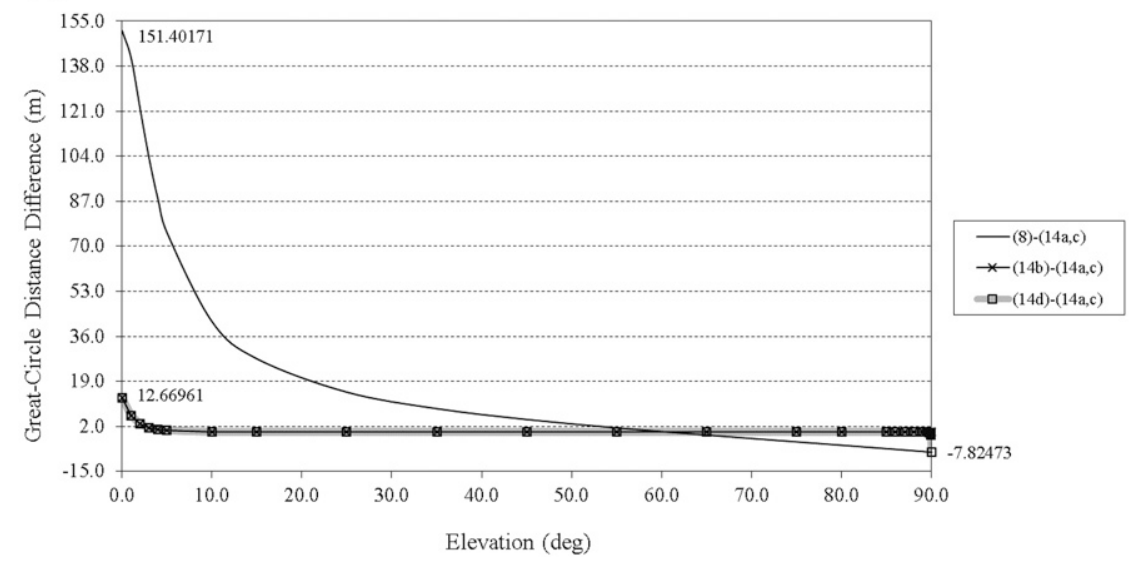

FIG. 2. Differences, as a function of elevation and at $h=20 \mathrm{~km}$ [computed using (13c)], between (a) $h$ and (b) $s$ values computed using the SECC and SEECC models. In (8) $h$ was computed using (7), in (14a) and (14c) $h$ was computed using (13a), in (14b) $h$ was computed using (13b), and in (14d) $h$ was computed using (13c). The parameter values are $h_{R_{-} \mathrm{MSL}}=h_{a}=$ $0 \mathrm{~km}, a=6370 \mathrm{~km}, r_{c}=4 a$, and $k_{e}=4 / 3$.

assumes that this gradient holds throughout the atmosphere ${ }^{4}$ and uses (6) with the simplifications following it applied to a $\theta=0^{\circ}$ ray, ray curvature under "typical" conditions is estimated as $r_{c}=4 a$. For the SEECC model, this corresponds to $k_{e}=4 / 3$ (Doviak and Zrnić 1993, section 2.2.3). In meteorology, typical propagation conditions are those that are most often assumed to hold when radar-centric coordinates are converted to Earth-centric coordinates. Of course, conditions can be far from typical, which can result in significant errors during coordinate transformations (e.g., Levis et al. 2010, chapter 6).

Because the SECC model is new, the first comparison involves this model and the SEECC model. Figure 2 shows $h$ and

\footnotetext{
${ }^{4}$ It is noted that the assumption that this gradient holds throughout the atmosphere results in $n$ values that become less than 1.0 at midtropospheric and higher altitudes. This, of course, is nonphysical.
}

$s$ differences between these models at an altitude of $20 \mathrm{~km}$ MSL [from (13c)]. This height was chosen because it approximately represents the upper limit of interest in (tropospheric) weather-radar applications and because differences (at a fixed elevation) between the various $h$ and $s$ formulations increase with increasing altitude (range). As shown in Fig. 2, the maximum height difference of $+8.70324 \mathrm{~m}$ is between (7) and (13a) at $0.0^{\circ}$ elevation, with other $0.0^{\circ}$ elevation height differences on the order of $1 \mathrm{~m}$. At $90.0^{\circ}$ elevation, all differences are less than the (7) and (13a) difference of $+0.00205 \mathrm{~m}$. For "typical" propagation conditions, therefore, the SECC height formulation (13a) is consistent with the SEECC formulation (7), and (13b) and (13c) are very accurate approximations of (13a).

As with the height formulations, differences between the great circle distance formulations at a fixed elevation increase with increasing altitude (range). In contrast with the respective 

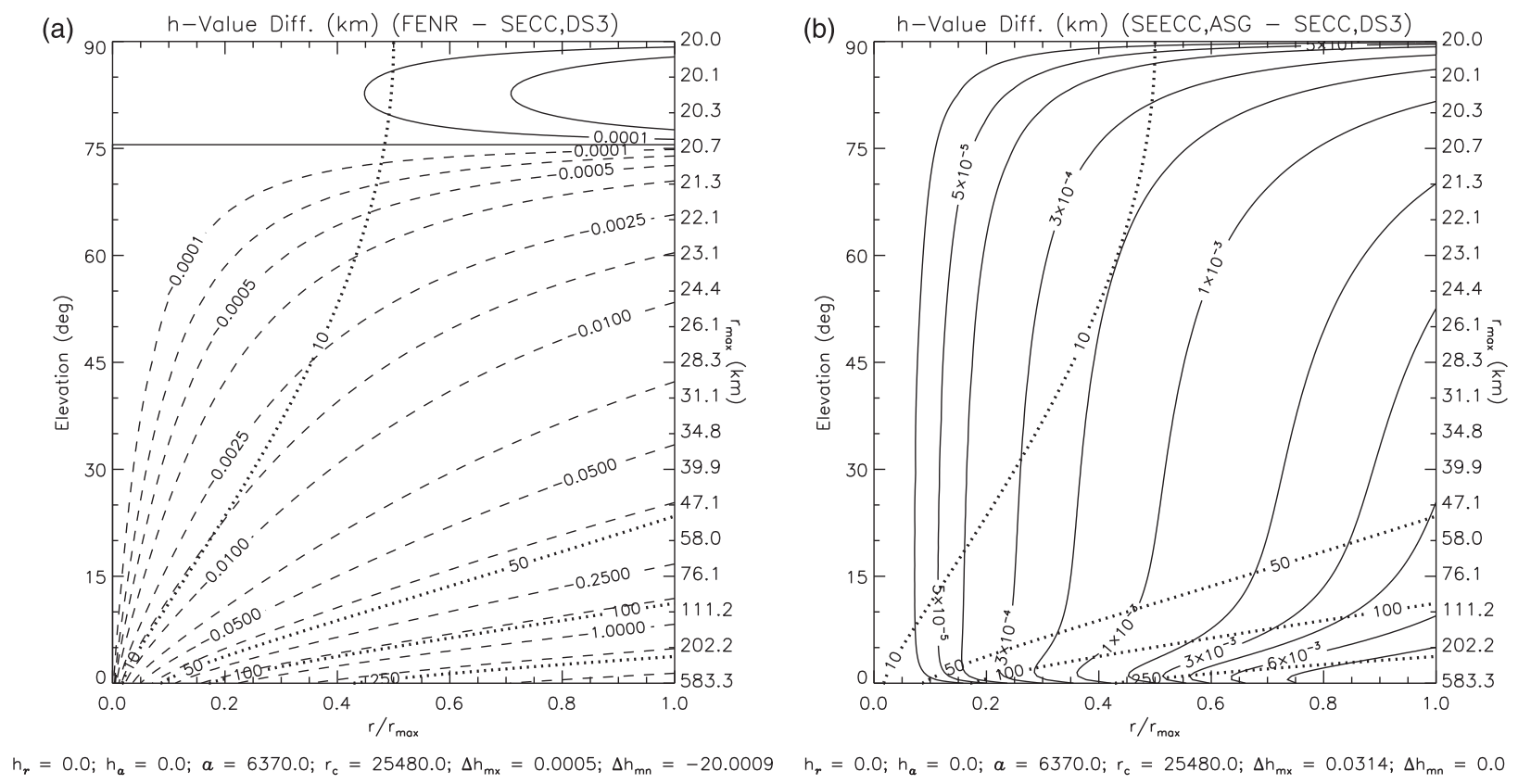

FIG. 3. (a) Differences between $h$ values $(\mathrm{km})$ obtained using the flat Earth no refraction [FENR; Eq. (1)] and the spherical Earth constant curvature, direct solution 3 [SECC,DS3; Eq. (13c)] models as a function of normalized range and elevation (see Table 2 for definitions of the acronyms that are used to identify propagation equations). (b) Differences between $h$ values (km) obtained using the spherical equivalent Earth constant curvature, approximate solution geometric [SEECC,ASG; Eq. (9)] and the SECC,DS3 [Eq. (13c)] models as a function of range and elevation. In both (a) and (b) the abscissa is normalized range $r / r_{\max }$, where $r_{\max }$ (values plotted on the right-hand ordinate axis) is the range that corresponds to a height $h$ of $20 \mathrm{~km}$ at the corresponding elevation (elevation values are plotted on the left-hand ordinate axis) or, if a height of $20 \mathrm{~km}$ is never reached, is either the range at which the ray intercepts the ground (assuming flat terrain) or the maximum range for which values were computed $(600 \mathrm{~km})$. Values are plotted as a function of normalized range in order to maximize conveyance of information regarding differences at altitudes below $20 \mathrm{~km}$. Positive height difference contours are indicated by solid lines, negative height difference contours are indicated by dashed lines, and nonconstant contour intervals are used to maximize conveyance of the spatial structure of difference fields. Constant range contours (at 10, 50, 100, and $250 \mathrm{~km})$ are indicated by thick-dotted gray lines. Values of $r_{\max }$ are computed using (16) when the "truth" equation [that on the rhs being subtracted from the test equation, which is on the lhs; in this figure, this is SECC,DS3, (13c)] is either (16) or (22) and are computed using (13a) otherwise except for $\theta=90^{\circ}$, where (13c) is used owing to its satisfying the no-refraction condition. The parameters are $h_{R \_\mathrm{MSL}}=h_{a}=0.0 \mathrm{~km}, a=6370.0 \mathrm{~km}$, $r_{c}=4 a=25480 \mathrm{~km}$, and $k_{e}=4 / 3$.

height formulations, the SEECC (8) and SECC (14a), (14c) formulations differ by what may be considered to be a significant amount for some elevations. As shown in Fig. 2b, at an altitude of $20 \mathrm{~km}$ the difference between (8) and (14a), (14c) ranges from $+151.40171 \mathrm{~m}$ at $0.0^{\circ}$ elevation to $-7.82473 \mathrm{~m}$ at $90.0^{\circ}$ elevation. For elevations greater than about $8.2^{\circ}$ (and an altitude of $20 \mathrm{~km}$ ), the magnitude of the difference between (8) and (14a), (14c) is less than about $50 \mathrm{~m}$. Differences between (14b), (14d) and (14a), (14c), on the other hand, are much smaller, being bracketed by $+12.66961 \mathrm{~m}$ and $-7.82473 \mathrm{~m}$ at $0.0^{\circ}$ and $90.0^{\circ}$ elevation, respectively. Thus, for typical refractive conditions the SECC great circle distance formulation (14a), (14c) contains some significant differences relative to the SEECC formulation (8). These differences are reflected in the SECC approximations (14b), (14d) since they are close approximations to $(14 \mathrm{a}),(14 \mathrm{c})$.

The SEECC model differs from the SECC model in that it utilizes a spatial transformation. As indicated by the results shown Fig. 2, this spatial transformation results in $h$ and $s$ values being changed slightly relative to the actual constant curvature values, which are given by (13a) and (14a), (14c) or [15a(1)] when $\theta \neq \pm 90.0^{\circ}$ and (13c) and (14d) [with $h$ in (14d) computed using (13c)] when $\theta= \pm 90.0^{\circ}$. In fact, the value of $k_{e}$ used in (7) has been varied to determine if the SEECC (7) and SECC (13a) formulations can be brought into perfect agreement. They cannot.

Differences between (1) and (13c) (FENR vs SECC) are shown in Fig. 3a (Table 2 provides definitions of the acronyms that are used to identify propagation equations). At low $\left(<15^{\circ}\right)$ elevations and moderate to large $(>50 \mathrm{~km})$ ranges, these differences are relatively large. At $\theta=0^{\circ}$, the FENR model (1) provides unsavory results (e.g., $20 \mathrm{~km}$ too low at $0^{\circ}$ elevation and a range of $583.3 \mathrm{~km}$ ) since it indicates that data this elevation are located at radar ground level. With the assumed typical refractive conditions, however, the curvature of Earth's surface results in data with $\theta=0^{\circ}$ being located above radar ground level.

Contrarily, (9) (SEECC Taylor series approximation for h) correctly indicates that data collected at $\theta=0^{\circ}$ are located above radar ground level. Equation (9) provides a fairly 
TABLE 2. Definitions of acronyms used to identify propagation equations. Acronym sets are structured according to the assumed Earth condition, ray assumption, and equation classification (e.g., SECC,DS3).

\begin{tabular}{lr}
\hline \hline \multicolumn{1}{c}{ Earth condition } & Ray assumption \\
\hline $\mathrm{FE}=$ flat Earth & NR $=$ no refraction \\
$\mathrm{SE}=$ spherical Earth & CC = constant curvature \\
$\mathrm{SEE}=$ spherical equivalent Earth &
\end{tabular}

Equation classification

AS $=$ approximate solution (equivalent Earth model) ${ }^{\mathrm{a}}$

ASG = approximate solution geometric (Bent et al. 1950)

DS1 = direct solution 1 [(13a), (14a), (14c), (15a)]

DS2 $=$ direct solution $2[(13 b),(14 b)]$

DS3 = direct solution 3 [(13c), (14d)]

DSTSA2 = direct solution Taylor series approximation second order [(20), (22)]

DS3TSA3 = direct solution 3 Taylor series approximation third order [(21), (23)]

RayTracing $=$ results obtained using the integral ray tracing approach

${ }^{a}$ The equivalent Earth model is labeled as an approximate solution because it utilizes a spatial transformation that, as indicated by the results shown in Fig. 2, results in $h$ and $s$ values being changed (slightly) relative to their actual values, which are given by (13a) and (14a), (14c) or (15a) when $\theta \neq \pm 90.0^{\circ}$ and (13c) and (14d) [with $h$ in (14d) computed using (13c)] when $\theta= \pm 90.0^{\circ}$.

accurate approximation of (13c), although it does produce values that are up to $31.4 \mathrm{~m}$ too large at $0^{\circ}$ elevation and a range of $583.3 \mathrm{~km}$ ( $h=20 \mathrm{~km}$ ) (Fig. 3b). Considering that, from Fig. 2, (7) (SEECC) produces a height that is $\sim 7.8 \mathrm{~m}$ greater than that produced by (13c) (SECC) at $0^{\circ}$ elevation and a range of $583.3 \mathrm{~km}(h=20 \mathrm{~km})$, it is apparent that the approximation (9) produces heights that are within $\sim 23.6 \mathrm{~m}$ of (7) for typical propagation conditions and for $h \leq 20 \mathrm{~km}$.

It is informative to explore the performance of (7) and (8) (SEECC), (10) and (11) (FECC), and (13c) and (14d) (SECC) relative to results obtained using ray tracing for two different reference $n$ profiles:

1) a linear- $n$ profile in which $d n / d h=-1 / 4 a$ and, thus, from (6) for which $r_{c} \approx 4 a$, and

2) an exponential- $n$ profile in which $n=313 \exp [-0.143859 h] / 10^{6}+$ 1 , which is an accurate representation of the average atmospheric $n$ structure in the United States and produces total bending (refraction) of rays that is less than $5 \%$ different from average bending conditions in the United States up to $h=20 \mathrm{~km}$ (Bean and Dutton 1966, section 3.8).

The ray tracing model used for these comparisons is described in appendix A and has been verified through comparison with an independently developed ray tracing model [the constant Earth radius model of Johnson (2010)]. Differences between values obtained using (7), (8), (13c), and (14d) and those obtained using ray tracing for the linear- $n$ atmosphere are shown in Fig. 4. The first thing to note from Fig. 4 is that height and ground range differences are not equivalently zero even though this test atmosphere should result in constant curvature propagation that is consistent with the curvature values used in (7), (8), (13c), and (14d). There are numerous reasons for nonzero difference values: 1) $\left\{1 /\left[n^{2}(h)\right]\right\}\left\{1 /\left[1+\left(h_{R_{-} \mathrm{MSL}}+h\right) / a\right]\right\}$ in (6) changes with height, which results in ray curvature changing with height; 2) because of the $\cos \left[\theta\left(h_{0}\right)\right]$ term in (6) ray curvature changes with initial elevation angle, which is not incorporated in these comparisons; and 3 ) the spatial transformation in the equivalent Earth model results in $h$ and $s$ values that differ slightly relative to their actual values. Despite these issues, the SECC model developed herein produces results that are very close to those obtained using ray tracing, with $h$ values within $\sim 4 \mathrm{~m}$ and $s$ values within $\sim 13 \mathrm{~m}$ of the values obtained using ray tracing. The SEECC model, on the other hand, produces $h$ values within $\sim 5 \mathrm{~m}$ of the ray tracing results but produces $s$ values that are up to $151 \mathrm{~m}$ too large, which is consistent with the results presented in Fig. 2.

Results for an exponential- $n$ atmosphere are shown in Fig. 5. The $h$-difference results for both the SECC and SEECC models are similar, with maximum $h$ differences relative to ray tracing results of approximately $74 \mathrm{~m}$ and minimum $h$ differences of approximately $-1400 \mathrm{~m}$. The $s$-difference results between these two models, however, are different. The SECC model produces a maximum $s$ difference of approximately $116 \mathrm{~m}$ and a minimum $s$ difference of approximately $-1.5 \mathrm{~m}$. The SEECC model, on the other hand, always produces $s$ values that are too large and produces a maximum $s$ difference of approximately $255 \mathrm{~m}$. Thus, for a reference exponential- $n$ atmosphere, the SECC model is more accurate than the SEECC model. Even so, both models produce results that are significantly different from truth (ray tracing), especially at low elevations.

Figure 6 provides results for the FECC model. For the linear $n$ profile, the FECC height Eq. (10) performs well while the great circle distance formulation (11) engenders much greater error (maximum of $+608 \mathrm{~m}$ ) at low elevations relative to the SECC and SEECC models (maximum errors of $+13 \mathrm{~m}$ and $+152 \mathrm{~m}$, respectively). For the exponential $n$ profile, performance of the FECC height relation is similar to that of the SECC and SEECC height relations while the great circle distance relation again engenders more error (maximum of $+705 \mathrm{~m}$ vs $+116 \mathrm{~m}$ and $+255 \mathrm{~m}$ for the SECC and SEECC models, respectively).

\section{b. Superrefraction}

It is useful to know how well the equations that are recommended for use under "typical" propagation conditions 


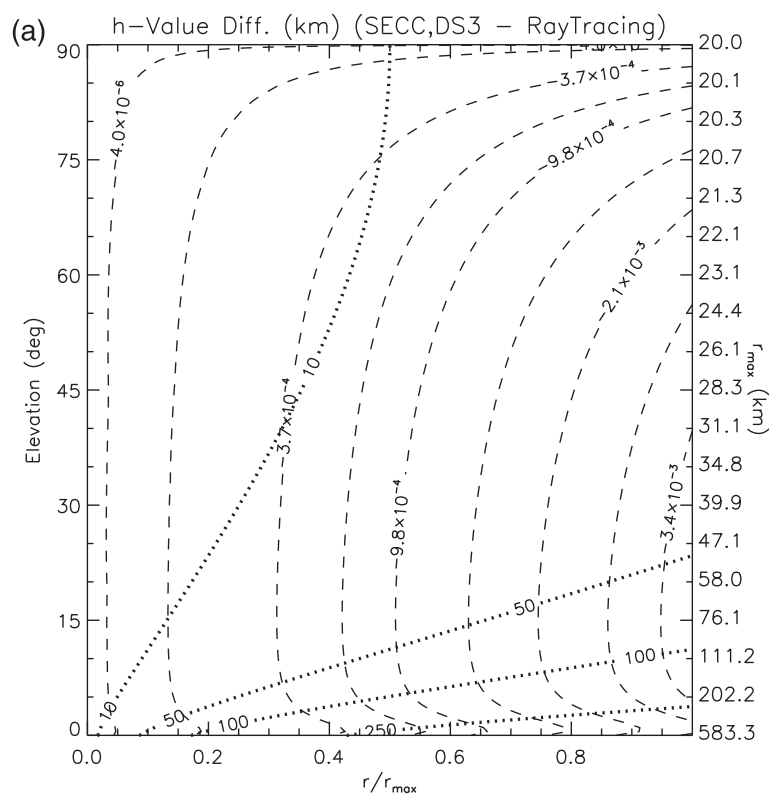

$h_{r}=0.0 ; h_{a}=0.0 ; a=6370.0 ; r_{c}=25480.0 ; \Delta h_{m x}=0.0 ; \Delta h_{m n}=-0.00379$

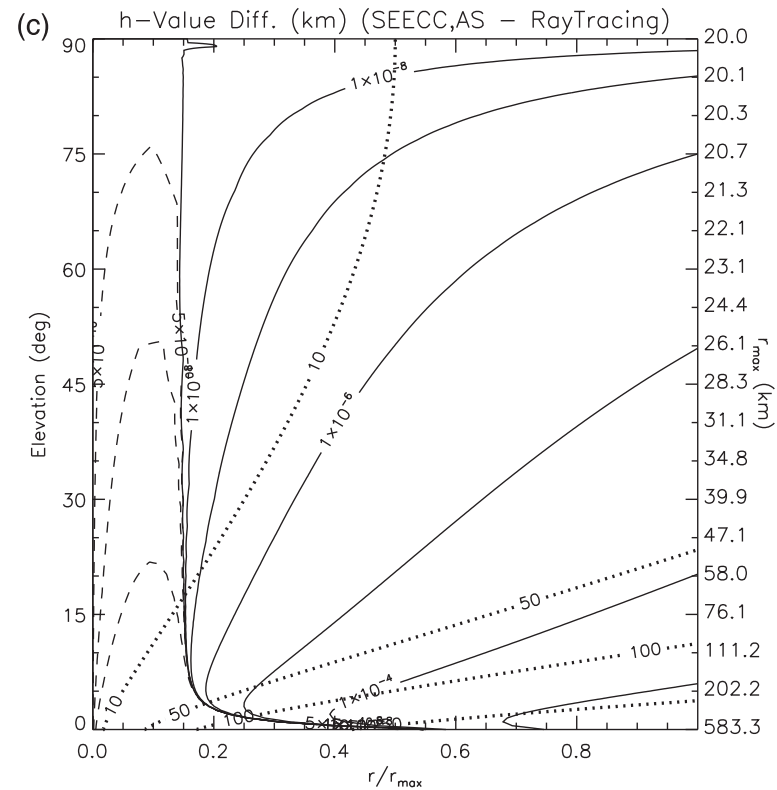

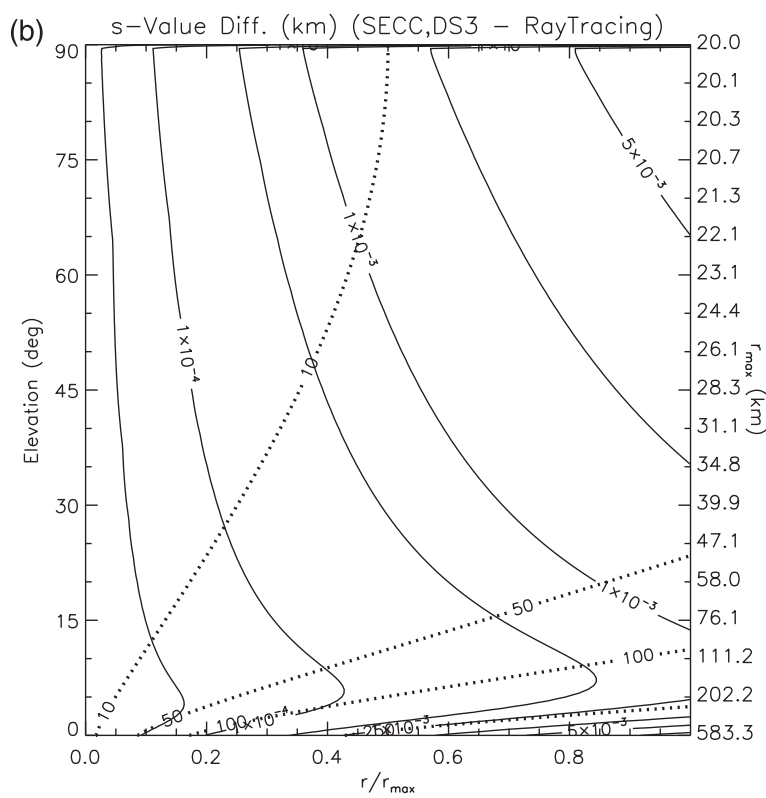

$\mathrm{h}_{r}=0.0 ; \mathrm{h}_{a}=0.0 ; a=6370.0 ; \mathrm{r}_{\mathrm{c}}=25480.0 ; \Delta \mathrm{s}_{\mathrm{mx}}=0.01296 ; \Delta \mathrm{s}_{\mathrm{mn}}=0.0$

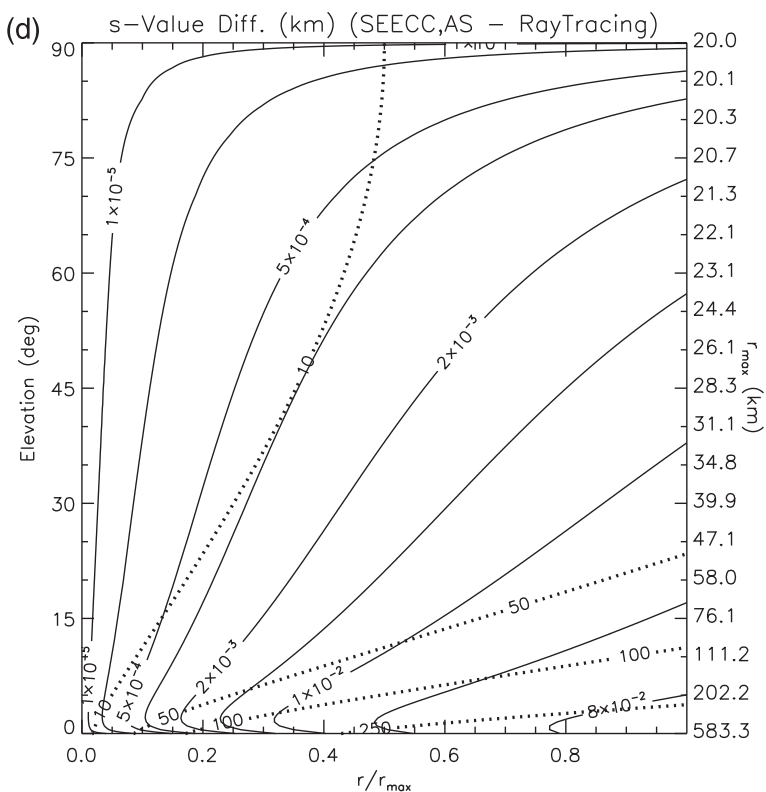

$\mathrm{h}_{r}=0.0 ; \mathrm{h}_{a}=0.0 ; a=6370.0 ; \mathrm{r}_{\mathrm{c}}=25480.0 ; \Delta \mathrm{h}_{\mathrm{mx}}=0.005 ; \Delta \mathrm{h}_{\mathrm{mn}}=-0.00016$

$h_{r}=0.0 ; h_{a}=0.0 ; a=6370.0 ; r_{c}=25480.0 ; \Delta s_{m x}=0.1517 ; \Delta s_{m n}=0.0$

FIG. 4. As in Fig. 3, except differences are for a linear- $n$ atmosphere in which $d n / d h=-1 / 4 a$ and are (a) between $h$ values computed using the SECC,DS3 [Eq. (13c)] model and ray tracing (RayTracing; appendix A); (b) between $s$ values computed using the SECC,DS3 [Eq. (14d) with $h$ computed using (13c)] model and RayTracing (appendix A); (c) between $h$ values computed using the spherical equivalent Earth constant curvature, approximate solution [SEECC,AS; Eq. (7)] model and RayTracing (appendix A); and (d) between $s$ values computed using the SEECC,AS [Eq. (8) with $h$ computed using (7)] model and RayTracing (appendix A). Normalized range values for these $h$ and $s$ difference plots are determined in the same manner as for the $h$ difference plots in Fig. 3. All differences have units of $\mathrm{km}$.

[(13c) and (14d); SECC] perform when conditions are not typical. To test this, results obtained using (13c) and (14d) are compared to those obtained using the full-solution Eqs. (13a) and (14a), (14c)/(15a) (SECC). For superrefraction, an atmosphere in which $d n / d h=-533 \times 10^{-6}$ is used, which is a fairly extreme superrefractive environment that was observed $13 \%-$ $27 \%$ of the time during the summer months of 2004 and 2005 in the British Channel Islands (Gunashekar et al. 2007). This environment produces an $r_{c}$ that is $\sim 1 / 13.6$ of its value under "typical" refractive conditions. It is noted that Gunashekar et al. (2007) 

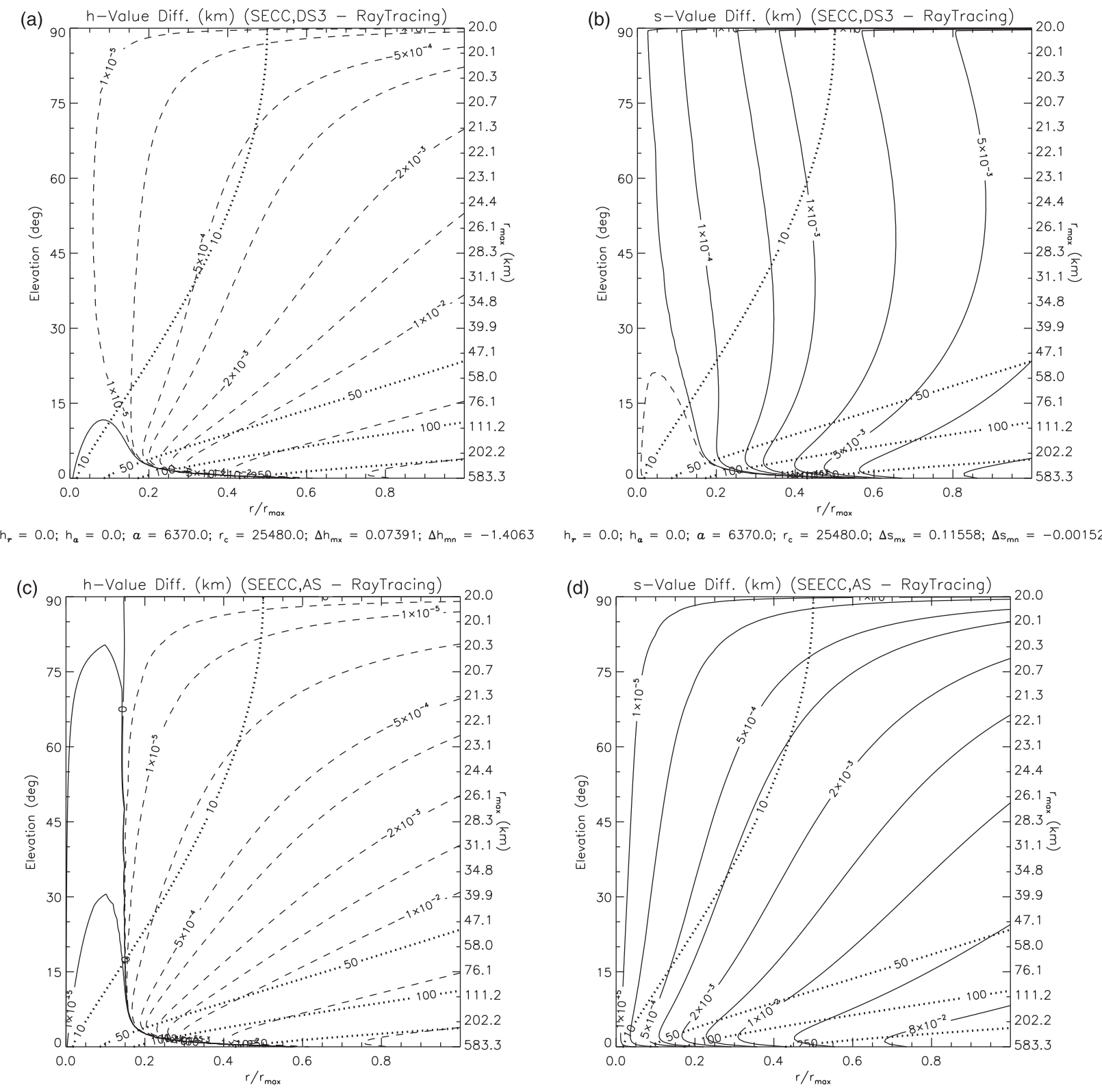

$h_{r}=0.0 ; h_{a}=0.0 ; a=6370.0 ; r_{c}=25480.0 ; \Delta h_{m x}=0.0741 ; \Delta h_{m n}=-1.3987$

$h_{r}=0.0 ; h_{a}=0.0 ; a=6370.0 ; r_{c}=25480.0 ; \Delta s_{m x}=0.25524 ; \Delta s_{m n}=0.0$

FIG. 5. As in Fig. 4, but for an exponential- $n$ atmosphere in which $n$ is given by $n=313 \exp [-0.143859 h] / 10^{6}+1$.

estimated $d n / d h$ using surface observations obtained at varying locations/altitudes. Consequently, their estimates of $d n / d h$ potentially incorporate a significant amount of error. Nonetheless, their observations do suggest that such gradients are possible.

The results shown in Fig. 7 indicate that for large ranges, which are present in Fig. 7 at elevations near $5^{\circ}$, differences between (13c) and (13a) and between (14d) and (14a), (14c)/(15a) can be significant. The maximum $h$ difference in Fig. 7a is approximately $671 \mathrm{~m}$ and the maximum $s$ difference in Fig. $7 \mathrm{~b}$ is approximately $2528 \mathrm{~m}$. In addition, at higher elevations $s$ differences are negative and become more so with increasing range, with the greatest difference of approximately $-106 \mathrm{~m}$.

\section{c. Strong subrefraction (negative radius of curvature)}

In this test $r_{c}$ has the same magnitude as that used in the superrefractive test shown in Fig. 7 but has the opposite sign, and, thus, a different sense of concavity relative to typical and superrefractive conditions. This $r_{c}$ value was used here as data regarding extreme subrefractive conditions are not readily available and it is postulated that if downward bending of this degree is possible then upward bending of this degree might also be possible. It is noted, however, that because downward bending is what occurs under "typical" conditions, the conditions for this test may be too extreme.

As shown in Fig. 8, maximum differences between (13c) and (13a) and between (14d) and (14a), (14c)/(15a) are much smaller 

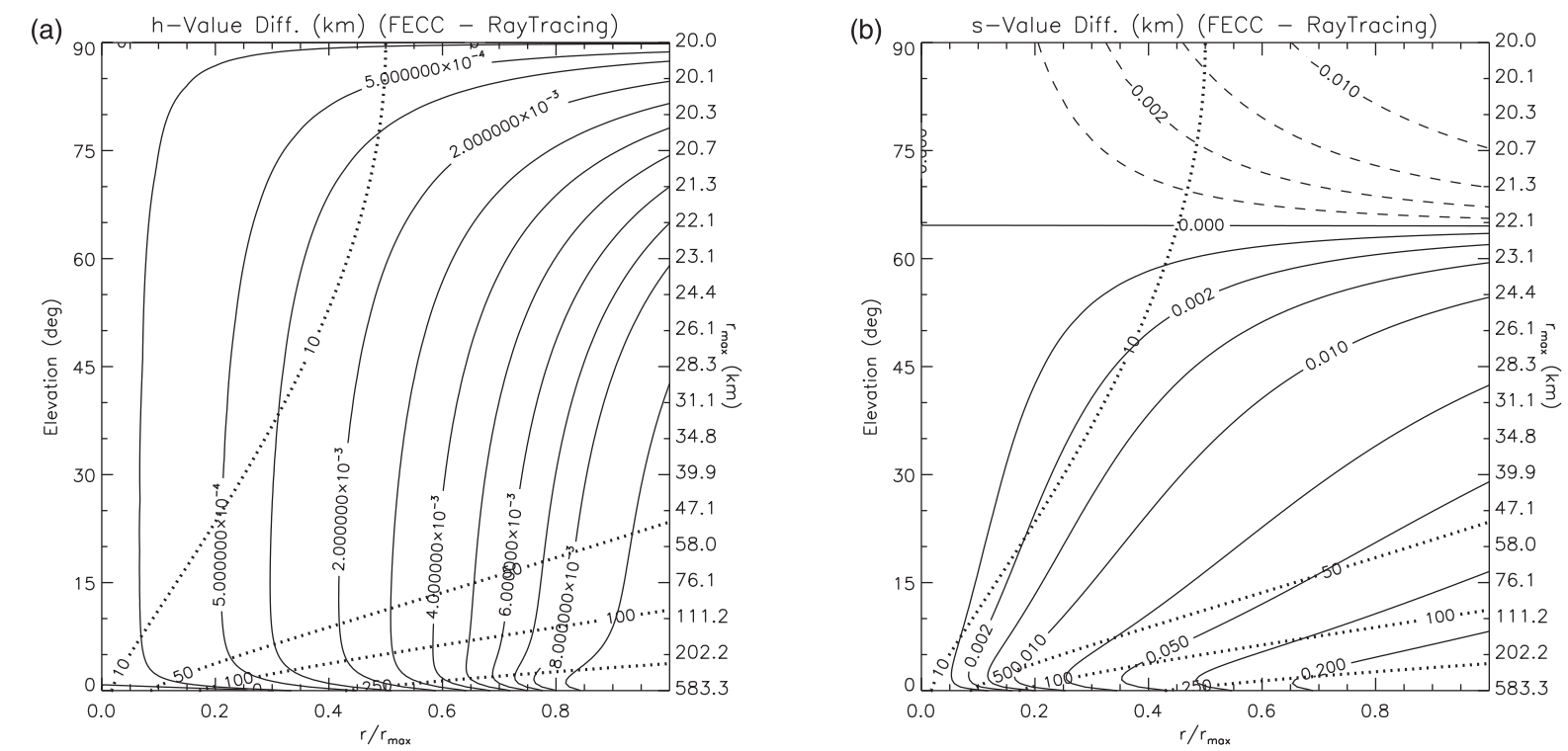

$h_{r}=0.0 ; h_{a}=0.0 ; a=6370.0 ; r_{c}=25480.0 ; \Delta h_{m x}=0.02057 ; \Delta h_{m n}=-5.00000 e-05$

$h_{r}=0.0 ; h_{a}=0.0 ; a=6370.0 ; r_{c}=25480.0 ; \Delta s_{m x}=0.60784 ; \Delta s_{m n}=-0.02355$
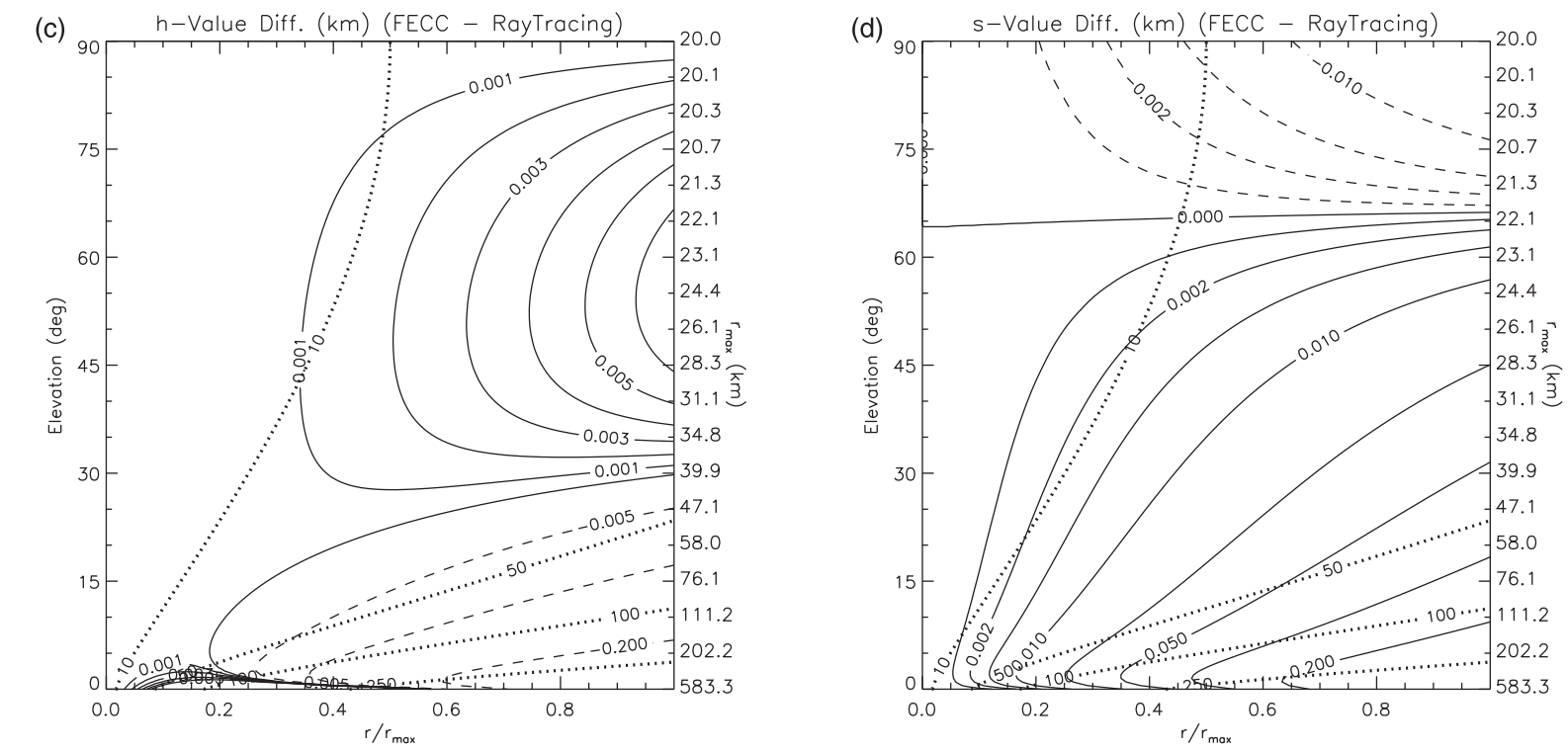

$h_{r}=0.0 ; h_{a}=0.0 ; a=6370.0 ; r_{c}=25480.0 ; \Delta h_{m x}=0.07449 ; \Delta h_{m n}=-1.38321$

$h_{r}=0.0 ; h_{a}=0.0 ; a=6370.0 ; r_{c}=25480.0 ; \Delta s_{m x}=0.70502 ; \Delta s_{m n}=-0.02355$

FIG. 6. As in Fig. 4, except differences are between the flat Earth constant curvature (FECC) model and ray tracing for (a),(b) a linear- $n$ atmosphere in which $d n / d h=-1 / 4 a$ and (c),(d) an exponential- $n$ atmosphere in which $n$ is given by $n=313 \exp [-0.143859 h] / 10^{6}+1$. Rays are assumed to have constant radius of curvature given by $r_{c f}=1 /(1 / 4 a-1 / a)=-(4 / 3 a)$.

than those for superrefractive conditions. The primary reason for this is the ranges in Fig. 8 are smaller than those in Fig. 7 because at each elevation the maximum range to which differences are computed $r_{\max }^{5}$ is reached at smaller values of $r$ for subrefraction

\footnotetext{
${ }^{5}$ The maximum range $r_{\max }$ is, for a given elevation, the range that corresponds to a height $h$ of $20 \mathrm{~km}$ or, if a height of $20 \mathrm{~km}$ is never reached, is either the range at which the ray intercepts the ground (assuming flat terrain) or the maximum range for which values were computed $(600 \mathrm{~km})$.
}

than for superrefraction. Because of this, $(13 \mathrm{c})$ is within $\sim 28 \mathrm{~m}$ of (13a) and (14d) is within $\sim 164 \mathrm{~m}$ of $(14 \mathrm{a}),(14 \mathrm{c}) /(15 \mathrm{a})$.

\section{d. Taylor series approximations}

For typical refractive conditions, the approximation (20) (SECC Taylor series approximation for $h$ ), with terms up to second order retained, performs quite well relative to (13a)-(13c) (SECC) as it produces values that are within $\sim 31.5 \mathrm{~m}$ of (13c) (Fig. 9a). The approximation (21) (SECC Taylor series approximation for $s$ ), however, does not perform as well as at low elevations and large ranges as it 

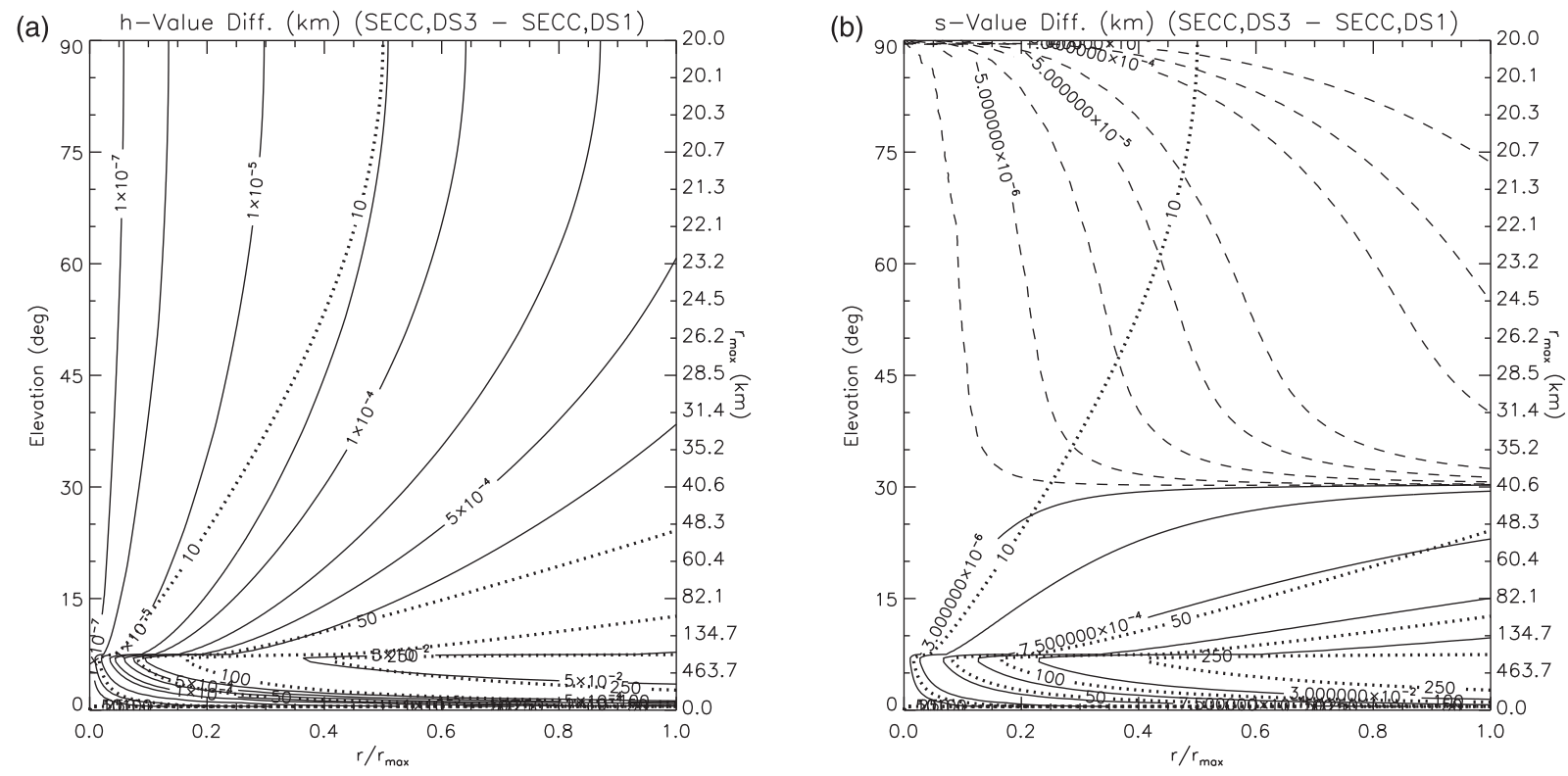

$h_{r}=0.0 ; h_{a}=0.0 ; a=6370.0 ; r_{c}=1876.17 ; \Delta h_{m x}=0.67061 ; \Delta h_{m n}=0.0$

$h_{r}=0.0 ; h_{a}=0.0 ; a=6370.0 ; r_{c}=1876.17 ; \Delta s_{m x}=2.5283 ; \Delta s_{m n}=-0.10627$

FIG. 7. As in Fig. 3, except differences are between (a) $h$ and (b) $s$ values obtained using the SECC,DS3 [Eqs. (13c) and (14d)] and the spherical Earth constant curvature, direct solution 1 [SECC,DS1; Eqs. (13a) and (14a), (14c)/(15a)] equations for a superrefractive atmosphere in which $r_{c}$ is $\sim 1 / 13.6$ of its value under "typical" refractive conditions. At $0.0^{\circ}$ elevation, $r_{\max }=0.0 \mathrm{~km}$ because rays emanating from this elevation propagate downward and intersect Earth's surface at $r=0.0 \mathrm{~km}$ since $h_{a}=0.0 \mathrm{~km}$.

produces values that are up to $\sim 1053 \mathrm{~m}$ larger than $(14 \mathrm{~d})$ (SECC). Interestingly, a comparison of Figs. 9a and 3b shows that (20) and (9) (SEECC Taylor series approximation for h) both produce maximum differences, for typical propagation conditions and relative to (13c), of $\sim 31.5 \mathrm{~m}$. Moreover, for low elevations the structure of the differences between $(20)$ and $(9)$ relative to (13c) are quite similar. Consequently, for "typical" propagation conditions (20) and (9) provide

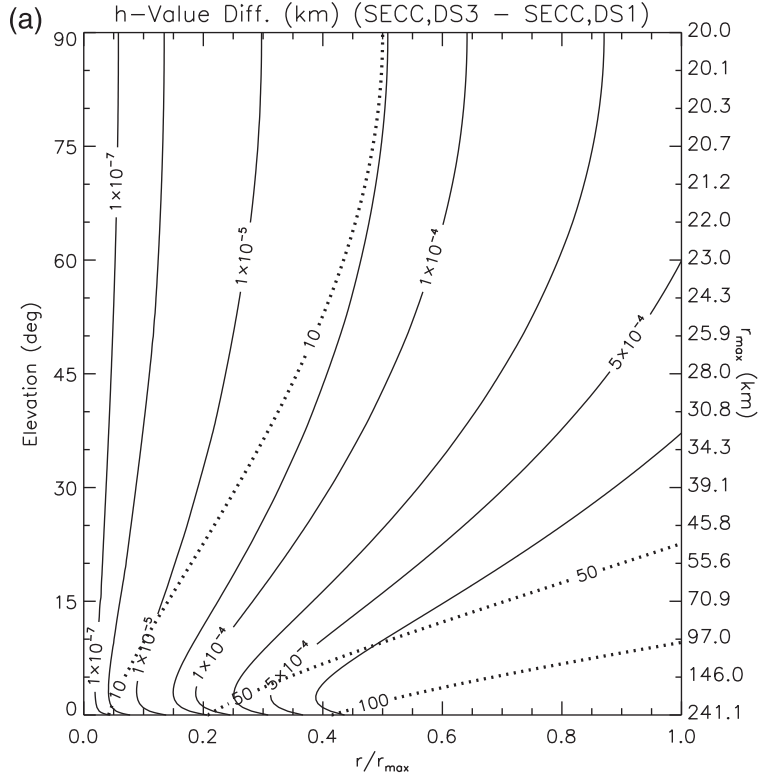

$\mathrm{h}_{r}=0.0 ; \mathrm{h}_{a}=0.0 ; a=6370.0 ; \mathrm{r}_{\mathrm{c}}=-1876.17 ; \Delta \mathrm{h}_{\mathrm{mx}}=0.02751 ; \Delta \mathrm{h}_{\mathrm{mn}}=0.0$

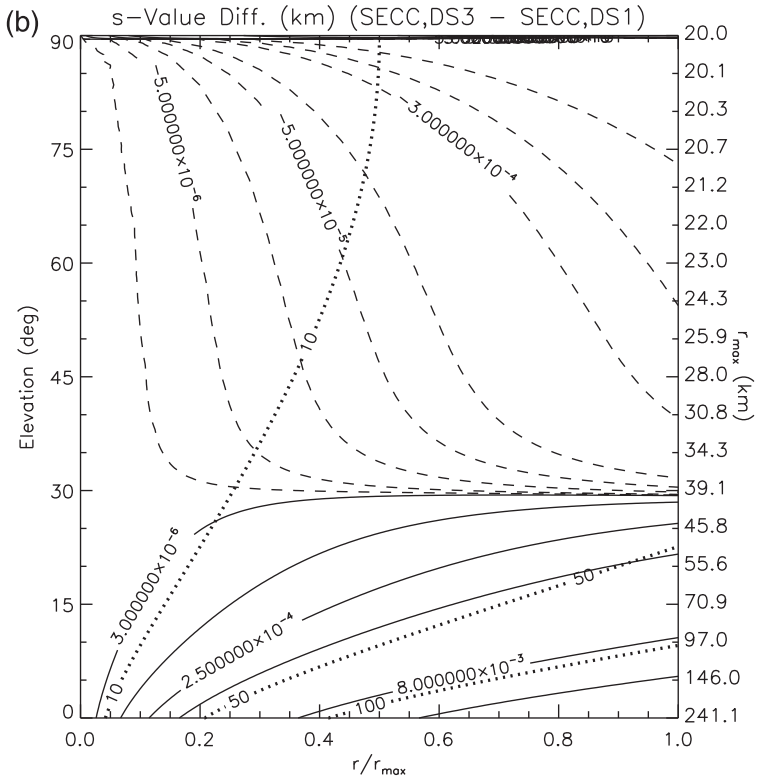

$h_{r}=0.0 ; h_{a}=0.0 ; a=6370.0 ; r_{c}=-1876.17 ; \Delta s_{m x}=0.16346 ; \Delta s_{m n}=-0.07114$

FIG. 8. As in Fig. 7, but for a subrefractive atmosphere in which $r_{c}$ has the same magnitude as that used in the superrefractive test shown in Fig. 7 but the opposite sign, and, thus, the opposite concavity relative to typical and superrefractive conditions. 

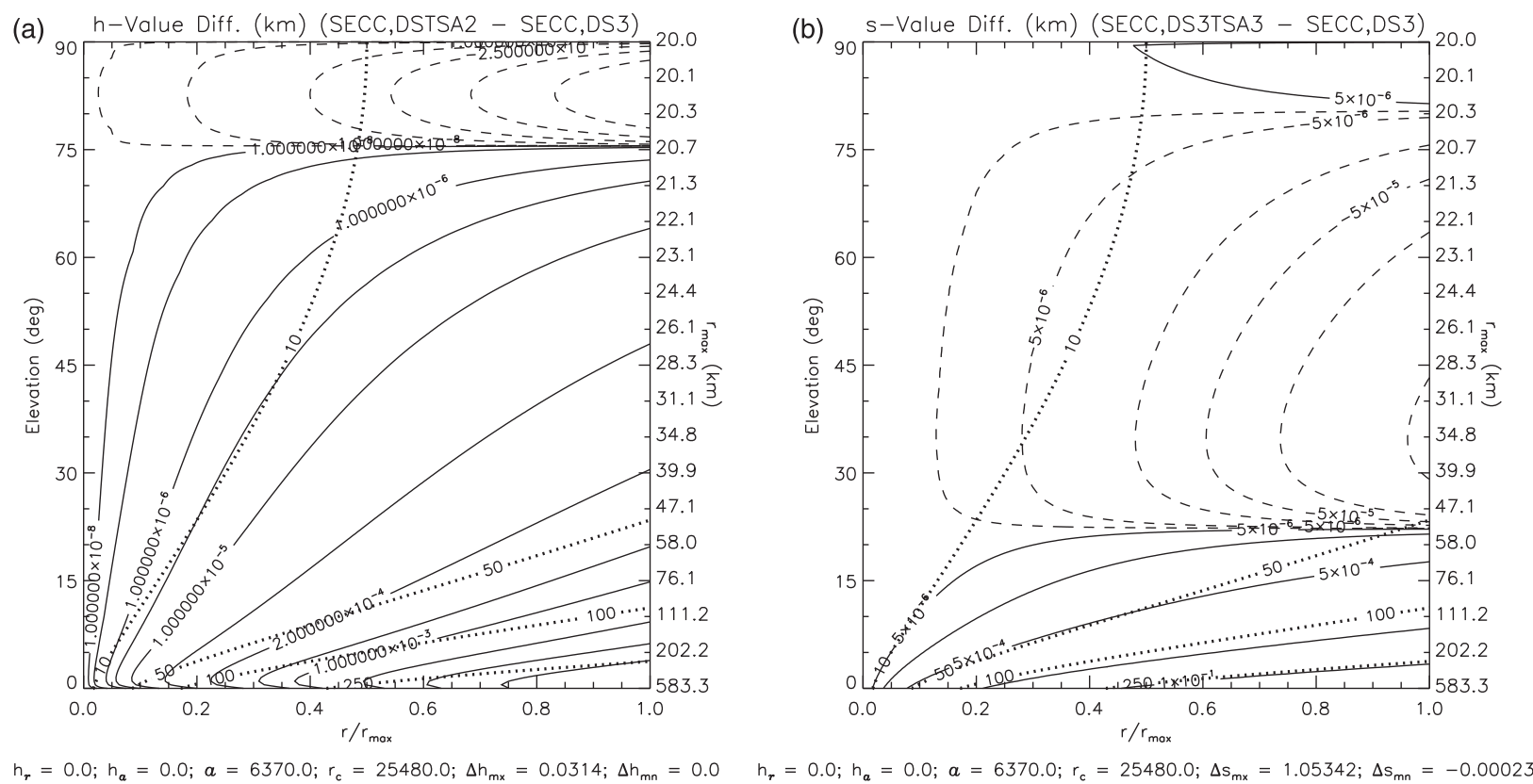

FIG. 9. As in Fig. 7, except 1) differences are for (a) $h$ values obtained using the spherical Earth constant curvature, direct solution Taylor series approximation second order [SECC,DSTSA2; Eq. (20)] and SECC,DS3 [Eq. (13c)] equations; and (b) $s$ values obtained using the spherical Earth constant curvature, direct solution 3 Taylor series approximation third order [SECC,DS3TSA3; Eq. (21)] and SECC,DS3 [Eq. (14d)] equations; and 2) values are for an atmosphere in which the ray radius of curvature is $r_{c}=4 a=25480 \mathrm{~km}$.

similar, but not equivalent, approximations to (13a)-(13c) (SECC) and (7) (SEECC).

For strong superrefraction and subrefraction (20) and (21) do not perform as well (Fig. 10). For strong superrefraction (20) has a maximum difference of $\sim 254 \mathrm{~m}$ relative to (13a), while for strong subrefraction (20) is within $\sim 59 \mathrm{~m}$ of $(13 \mathrm{a})$. The performance of (21) relative to $(14 a),(14 c) /(15 a)$ is much worse, as for strong superrefractive conditions the maximum difference is $\sim 2981 \mathrm{~m}$ for elevations near $5^{\circ}$ and large ranges and for strong subrefractive conditions the maximum difference is $\sim 1359 \mathrm{~m}$.

\section{e. Summary}

A summary of the performance of propagation equations tested herein is provided in Table 3 . The criteria used to classify equation performance as excellent $(E)$, good $(G)$, fair $(F)$, or poor $(\mathrm{P})$ are based upon the approximate length of a city block, which is $100 \mathrm{~m}$. If an $s$ equation error can be on the order of a city block or two its performance is said to be fair, if it is within a city block its performance is either good (greater than $50 \mathrm{~m}$ and less than $100 \mathrm{~m}$ ) or excellent (less than or equal to $50 \mathrm{~m}$ ), and if it is significant relative to a city block its performance is said to be poor. The length of a city block is used here because radar data are oftentimes displayed relative to population centers and the city block is the base organizational structure of such centers. The same criteria are used for $h$ equations, although one might argue that these criteria should be scaled. As such scaling depends upon the application at hand, it has not been applied here.
As shown in Table 3, the equations, with the exception of (1) (FENR), (8) (SEECC), (11) (FECC), and (21) (SECC Taylor series approximation for $s$ ), all performed excellent for "typical" propagation conditions. For strong superrefractive and subrefractive conditions, however, one should generally use the unaltered Eqs. (13a) and (14a), (14c)/(15a) (SECC). Finally, relative to a reference exponential atmosphere the performance of the SECC relations developed herein [(13c) and $(14 \mathrm{~d})]$ is better than that of the SEECC relations $[(7)$ and (8)] and the FECC relations [(10) and (11)], although for $h$ performance is poor for all of the simplified propagation models.

\section{Discussion}

Schelleng et al. (1933), who advanced the use of the SEECC model (Bean and Dutton 1966, p. 56), indicate that the spatial transformation utilized in this model results in negligibly small errors. This is further backed by Millington (1957), who showed that under this spatial transformation the relation between $h$ and $s$ remains the same out to second order. The tests performed herein support their conclusions, although for typical propagation conditions and $h \leq 20 \mathrm{~km}$, (8) (SEECC) does have errors on the order of a city block (Fig. 4d; Table 3 ).

Increasing the degree of the spatial transformation increases the associated errors. For the FECC model, the increase in $s$ errors is more impactful than for $h$, as maximum errors for typical propagation conditions grow to the order of several city blocks (Fig. 6b; Table 3).

One may argue that for typical propagation conditions the SECC equations derived herein [(13a)-(13c) (14a)-(14d), 


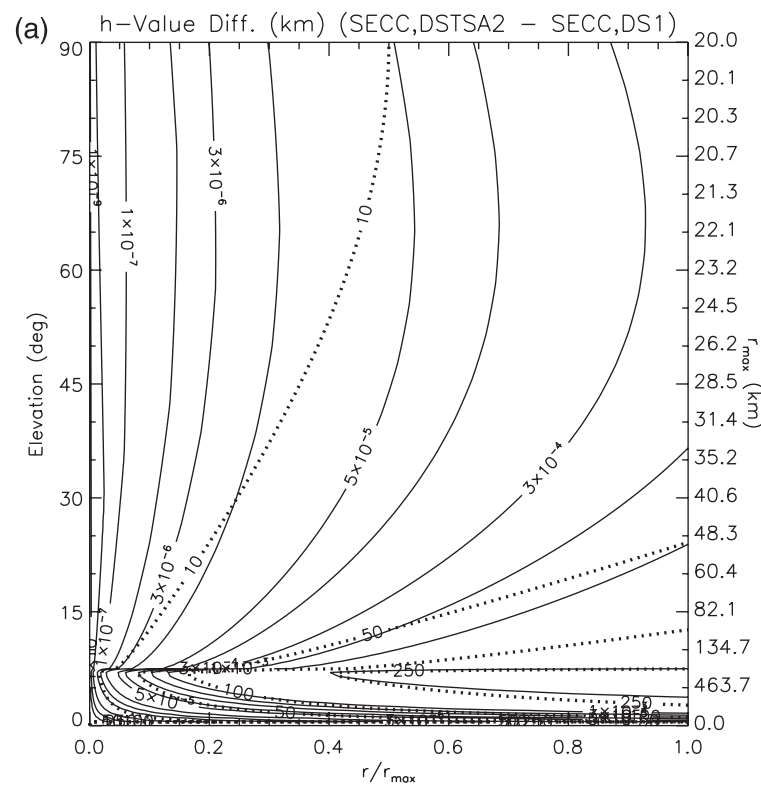

$h_{r}=0.0 ; h_{a}=0.0 ; a=6370.0 ; r_{c}=1876.17 ; \Delta h_{m x}=0.2539 ; \Delta h_{m n}=0.0$

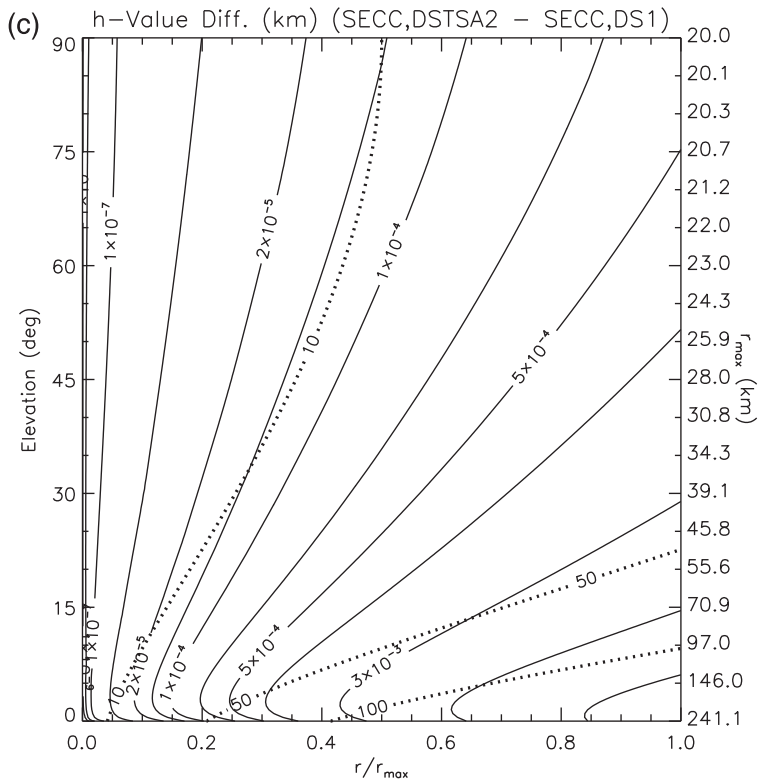

$h_{r}=0.0 ; h_{a}=0.0 ; a=6370.0 ; r_{c}=-1876.17 ; \Delta h_{m x}=0.05899 ; \Delta h_{m n}=0.0$

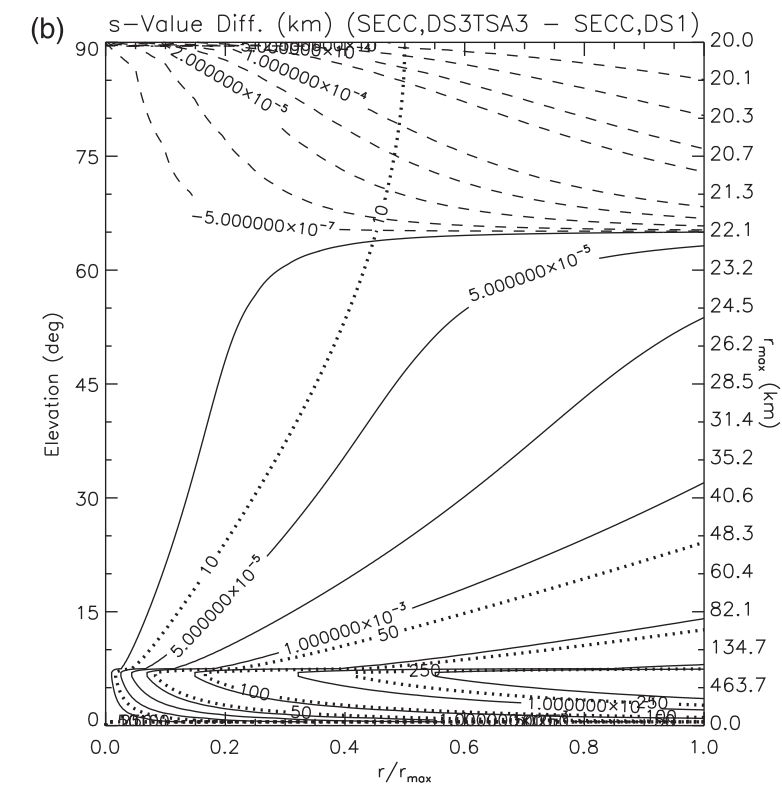

$h_{r}=0.0 ; h_{a}=0.0 ; a=6370.0 ; r_{c}=1876.17 ; \Delta s_{m x}=2.9807 ; \Delta s_{m n}=-0.10627$

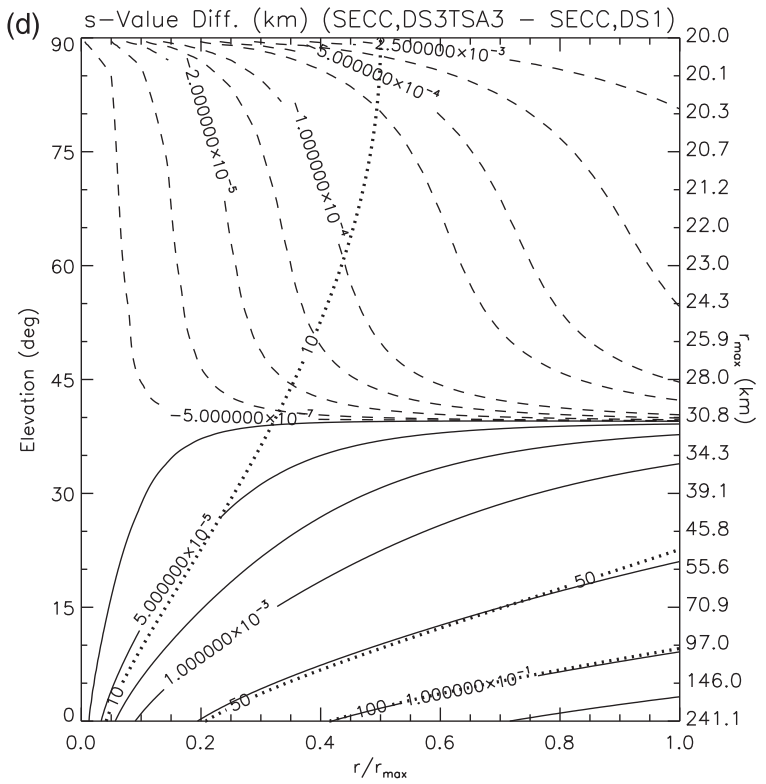

$h_{r}=0.0 ; h_{a}=0.0 ; a=6370.0 ; r_{c}=-1876.17 ; \Delta s_{m x}=1.35928 ; \Delta s_{m n}=-0.07114$

FIG. 10. As in Fig. 9, except 1) $h$ differences are between the SECC,DSTSA2 [Eq. (20)] and the SECC,DS1 [Eq. (13a)] equations; 2$) s$ differences are between the SECC,DS3TSA3 [Eq. (21)] and the SECC,DS1 [Eqs. (14a),(14c)/(15a)] equations; 3) differences in (a) and (b) are for a superrefractive atmosphere in which $r_{c}$ is $\sim 1 / 13.6$ of its value under "typical" refractive conditions (as in Fig. 7); 4) differences in (c) and (d) are for a subrefractive atmosphere having the same $r_{c}$ as that in Fig. 8.

and $(15 \mathrm{a})-(15 \mathrm{~b})]$ provide no additional value relative to the SEECC Eqs. (7) and (8) because of their close agreement. It can be argued, however, that the SECC equations derived herein are preferable to the SEECC equations since they do not require the slightly imperfect spatial transformation utilized in the SEECC model and are thus less complicated conceptually. Moreover, the equations derived herein provide the true constant-curvature solution.
The benefit of the simplified propagation model that is used, of course, depends upon the application and error tolerance. The FECC model, for instance, may be preferable for Doppler radar simulations (Davies-Jones et al. 2019), even though its error characteristics are not as good, for typical propagation conditions, as those of the SECC and SEECC models. This is especially true if error performance at relatively low elevations and large ranges is not important 
TABLE 3. A summary of the performance of propagation equations. Scoring criteria indicate the degree of maximum difference, in an absolute value sense, for elevations and ranges used in Figs. 2-10 (see the captions of Figs. 2 and 3 for more information regarding how the maximum range is determined) between the equation and the reference equation. Scores are defined to be $\mathrm{E}$ (excellent) $\leq 50 \mathrm{~m}, 50<\mathrm{G}$ (good) $\leq 100 \mathrm{~m}, 100<\mathrm{F}$ (fair) $\leq 200 \mathrm{~m}$, and $200 \mathrm{~m}<\mathrm{P}$ (poor). Values in parentheses are equation - reference equation differences (in $\mathrm{m}$ ), the absolute values of which are the maximum for the elevations and ranges used in Figs. 2-10. A dash indicates a test that would provide little useful information-it makes little sense to use (13c) as the reference equation for superrefractive conditions, for instance, unless the performance of (13c) is excellent for that condition, which it is not.

\begin{tabular}{|c|c|c|c|c|c|}
\hline \multirow[b]{2}{*}{ Equation; model } & \multirow[b]{2}{*}{ Reference equation; model } & \multicolumn{4}{|c|}{ Propagation environment } \\
\hline & & Typical & Superrefractive & Subrefractive & Exponential $n$ \\
\hline (7); SEECC & RayTracing & $E(5.0)$ & & & $P(-1398.7)$ \\
\hline (7); SEECC & (13a); SECC & $E(8.7)$ & & & \\
\hline$(13 \mathrm{c}) ; \mathrm{SECC}$ & RayTracing & $E(-3.8)$ & & & $P(-1406.3)$ \\
\hline (10); FECC & RayTracing & $E(20.6)$ & & & $P(-1383.2)$ \\
\hline (13c); SECC & (13a); SECC & $E(0.9)$ & $P(670.6)$ & $E(27.5)$ & \\
\hline (20); SECC & $(13 \mathrm{c}) ; \mathrm{SECC}$ & $E(31.4)$ & - & & - \\
\hline (20); SECC & (13a); SECC & & $P(253.9)$ & $G(59.0)$ & \\
\hline (1); FENR & (13c); SECC & $P(-20000.9)$ & - & & - \\
\hline (9); SEECC & (13c); SECC & $E(31.4)$ & - & & - \\
\hline (8); SEECC & RayTracing & $F(151.7)$ & & & $P(255.2)$ \\
\hline (8); SEECC & $(14 a),(14 c) /(15 a) ;$ SECC & $F(151.4)$ & & & \\
\hline$(14 d) ;$ SECC & RayTracing & $E(13.0)$ & & & $F(115.6)$ \\
\hline (11); FECC & RayTracing & $P(607.8)$ & & & $P(705.0)$ \\
\hline$(14 d) ;$ SECC & $(14 a),(14 c) /(15 a) ;$ SECC & $E(12.7)$ & $P(2528.3)$ & $F(163.5)$ & \\
\hline (21); SECC & $(14 d) ;$ SECC & $P(1053.4)$ & - & - & - \\
\hline (21); SECC & $(14 a),(14 c) /(15 a) ;$ SECC & & $P(2980.7)$ & $P(1359.3)$ & \\
\hline
\end{tabular}

to the application for which the simplified propagation model is being used.

As discussed earlier, for "typical" propagation conditions (13c) and (14d) (SECC) are recommended owing to their accuracy and their satisfaction of the no-refraction condition when $\theta= \pm 90.0^{\circ}$. However, when strong superrefraction or subrefraction occur (13a) and (14a),(14c) [or (15a)] should be used except when $\theta= \pm 90.0^{\circ}$, for which $h=h_{a} \pm r$ and $s=0$. A more accurate, and more complicated, approach would be to use (13a) and (14a), (14c) [or (15a)] and to vary $r_{c}$ in them using (6) while likely making the approximation $\left[n\left(h_{0}\right) / n^{2}(h)\right][(a+$ $\left.\left.h_{R_{-} \mathrm{MSL}}+h_{0}\right) /\left(a+h_{R_{-} \mathrm{MSL}}+h\right)\right] \approx 1$. The accuracy of such an approach will be tested in future work.

The Taylor series expansion (20) (SECC $h$; out to second order) performs very well under typical propagation conditions, as does the Bent et al. (1950) relation (9) (SEECC). The use of (20) is not recommended, however, when strong superrefraction or subrefraction occur. Moreover, the thirdorder Taylor series expansion (21) (SECC $s$ ) does not perform well for any conditions. It is noted that a Taylor series expansion of (14d) (SECC $s$ ) that retains terms up through fourth order in $r$ was tested. This nearly achieved a rating of fair $(\mathrm{F})$, with a greatest difference of approximately $-209 \mathrm{~m}$. This relation, however, contains numerous terms and thus does not provide any benefit/simplification relative to using (14d) itself.

The superrefractive and subrefractive tests conducted herein may be too severe since such extreme conditions are typically observed over relatively shallow layers in the atmosphere. Such extreme conditions, therefore, do not generally apply over large pathlengths, as they did in the tests performed herein, unless rays remain in those relatively shallow layers.
While this is true, the tests performed herein do provide bounds for performance under extreme conditions, which was the purpose of these tests.

Finally, the ubiquitous assumption in radar meteorology that Earth is spherical has been applied herein. Impacts of Earth's ellipticity are beyond the scope of this investigation. Errors associated with assumption of sphericity range up to $\sim 0.5 \%$ (e.g., Tseng and Lee 2010). Given this, it would be interesting to explore development of propagation relations for an elliptical Earth and errors for resulting formulations.

\section{Conclusions}

The purpose of this work was to present a new simplified propagation model (SECC) and provide practical guidance regarding errors associated with commonly used simplified propagation models. The following are the principal findings of this work:

1) Given the assumption that rays follow paths of constant curvature, a relatively simple set of propagation equations (SECC model) can be derived without invoking the spatial transformation that is utilized in the SEECC model.

2) With the assumption of constant radius of curvature $r_{c}$, the SECC model derived herein arises naturally from ray tracing relations.

3) Because the propagation equations derived herein do not invoke the slightly imperfect spatial transformation that is utilized in the SEECC and FECC models, these equations are more accurate than the SEECC and FECC equations for "typical" propagation conditions in which $d n / d h=-1 / 4 a$.

4) For a reference atmosphere in which $n=313 \exp [-0.143859 \mathrm{~h}] /$ $10^{6}+1$, which is a very accurate representation of the average 
atmospheric $n$ structure in the United States, the equations derived herein (SECC model) provide a more accurate solution than that provided by the SEECC and FECC equations, although all of these simplified propagation models produce relatively large height errors at low elevations and large ranges.

5) A Taylor series approximation for the height equations derived herein that performs well under typical propagation conditions and has an appearance that is similar to equations derived by Bent et al. (1950) and Gao et al. (2006) was obtained. A useful Taylor series approximation for great circle distance was not obtained.

Acknowledgments. The authors thank Dr. Mounir Chrit for his review of appendix B. This research was sponsored in part by the U.S. Air Force under Contract FA4861-07-R-C003 and in part by the National Science Foundation under Grants ATM-0003869 and ATM-0340693.

\section{APPENDIX A}

\section{The Integral Ray Tracing Model}

Given the condition $\lambda d n / d h \ll 1$, where $\lambda$ is wavelength, electromagnetic energy paths can be determined using ray theory (Stratton 1941, p. 343; Bean and Dutton 1966, section 3.2). When doing so, one may use numerous approaches. These are briefly categorized before details of the approach used herein are provided.

As illustrated by Zeng et al. (2014), second-order ordinary differential equations can be developed. Zeng et al. (2014) developed the relation

$$
\begin{gathered}
\frac{d^{2} h}{d r^{2}}+\left[\frac{1}{n} \frac{d n}{d h}+\frac{1}{\left(a+h_{R_{-} \mathrm{MSL}}+h\right)}\right]\left(\frac{d h}{d r}\right)^{2} \\
-\left[\frac{1}{n} \frac{d n}{d h}+\frac{1}{\left(a+h_{R_{-} \mathrm{MSL}}+h\right)}\right]=0 .
\end{gathered}
$$

Using the same approach, the following relations can be derived for the apparent range $r_{a}$, which results from assuming that electromagnetic energy propagates at the speed of light in a vacuum $c\left(r_{a}=\int_{r^{\prime}=0}^{r^{\prime}=r} n d r^{\prime}\right.$ and, thus, $\left.r_{a} \geq r\right)$, and great circle distance (at mean sea level) $s$

$$
\begin{aligned}
\frac{d^{2} h}{d r_{a}^{2}}+ & {\left[\frac{2}{n} \frac{d n}{d h}+\frac{1}{\left(a+h_{R \_\mathrm{MSL}}+h\right)}\right]\left(\frac{d h}{d r_{a}}\right)^{2} } \\
- & \frac{1}{n^{2}}\left[\frac{1}{n} \frac{d n}{d h}+\frac{1}{\left(a+h_{R_{-} \mathrm{MSL}}+h\right)}\right]=0
\end{aligned}
$$

and

$$
\begin{aligned}
\frac{d^{2} h}{d s^{2}}- & {\left[\frac{1}{n} \frac{d n}{d h}+\frac{2}{\left(a+h_{R_{-} \mathrm{MSL}}+h\right)}\right]\left(\frac{d h}{d s}\right)^{2} } \\
- & \left(\frac{a+h_{R_{-} \mathrm{MSL}}+h}{a}\right)^{2}\left[\frac{1}{n} \frac{d n}{d h}+\frac{1}{\left(a+h_{R_{-} \mathrm{MSL}}+h\right)}\right]=0 .
\end{aligned}
$$

The latter relation has been derived previously (Appleton 1946; Hartree et al. 1946). It is noted that these have the associated relations $d h / d r=d h / d r_{a}=d h / d s=0$ when $\theta=0$.

Because (A1)-(A3) are autonomous equations (no explicit dependence upon $r, r_{a}$, or $s$ ), the order of these equations can be reduced through the substitutions $w(h)=d h / d r, w(h)=$ $d h / d r_{a}$, and $w(h)=d h / d s$ (Polyanin and Zaitsev 2003, p. 34). Each of the resulting relations, through substitutions, can be expressed in terms of an Abel equation (Polyanin and Zaitsev 2003, p. 132). These Abel equations can be integrated, which produces

$$
\begin{aligned}
\frac{d r}{d h} & = \pm \frac{\left(a+h_{R_{-} \mathrm{MSL}}+h\right) n(h)}{\sqrt{\left(a+h_{R_{-} \mathrm{MSL}}+h\right)^{2} n^{2}(h)-C_{\mathrm{SL}}^{2}}}, \\
\frac{d r_{a}}{d h} & = \pm \frac{\left(a+h_{R_{-} \mathrm{MSL}}+h\right) n^{2}(h)}{\sqrt{\left(a+h_{R_{-} \mathrm{MSL}}+h\right)^{2} n^{2}(h)-C_{\mathrm{SL}}^{2}}},
\end{aligned}
$$

and

$$
\frac{d s}{d h}= \pm \frac{a C_{\mathrm{SL}}}{\left(a+h_{R_{-} \mathrm{MSL}}+h\right) \sqrt{\left(a+h_{R_{-} \mathrm{MSL}}+h\right)^{2} n^{2}(h)-C_{\mathrm{SL}}^{2}}},
$$

where $C_{\mathrm{SL}}=\left(a+h_{R \_\mathrm{MSL}}+h_{0}\right) n\left(h_{0}\right) \cos \left[\theta\left(h_{0}\right)\right]$ is a constant evaluated at the reference height $h_{0}$ (integration limit) that comes from Snell's law \{Snell's law of refraction for spherically stratified atmospheres is $\left(a+h_{R_{-} \mathrm{MSL}}+h\right) n(h) \cos [\theta(h)]=$ const and is also known as Bouger's, or Bouguer's, law; Bean and Dutton 1966, section 3.1; Kursinski et al. 2000; Coleman 2004\}. Equations (A4)-(A6) can be derived from geometrical considerations in a spherically stratified atmosphere (see, for instance, Doviak and Zrnić 1993, appendix A). In (A4)(A6), the positive signs apply for upward propagation ( $h$ increasing with increasing $r, r_{a}$, or $s$ ) and the negative signs apply for downward propagation ( $h$ decreasing with increasing $r, r_{a}$, or $s$ ).

In addition to solving first- and second-order ordinary differential equations, one may utilize a stepwise ray tracing technique (e.g., Johnson 2010; Zeng et al. 2014). Such techniques utilize a discretization of Snell's law.

Herein, the first-order ordinary differential equations, (A4)(A6), are solved. These relations can easily be integrated using separation of variables to produce

$$
r(h)=r\left(h_{k}\right) \pm \int_{h^{\prime}=h_{k}}^{h^{\prime}=h} \frac{\left(a+h_{R_{-} \mathrm{MSL}}+h^{\prime}\right) n\left(h^{\prime}\right)}{\sqrt{\left(a+h_{R_{-} \mathrm{MSL}}+h^{\prime}\right)^{2} n^{2}\left(h^{\prime}\right)-C_{\mathrm{SL}}^{2}}} d h^{\prime},
$$

$$
r_{a}(h)=r_{a}\left(h_{k}\right) \pm \int_{h^{\prime}=h_{k}}^{h^{\prime}=h} \frac{\left(a+h_{R_{-} \mathrm{MSL}}+h^{\prime}\right) n^{2}\left(h^{\prime}\right)}{\sqrt{\left(a+h_{R_{-} \mathrm{MSL}}+h^{\prime}\right)^{2} n^{2}\left(h^{\prime}\right)-C_{\mathrm{SL}}^{2}}} d h^{\prime} \text {, }
$$




$$
s(h)=s\left(h_{k}\right) \pm \int_{h^{\prime}=h_{k}}^{h^{\prime}=h} \frac{a C_{\mathrm{SL}}}{\left(a+h_{R_{-} \mathrm{MSL}}+h^{\prime}\right) \sqrt{\left(a+h_{R_{-} \mathrm{MSL}}+h^{\prime}\right)^{2} n^{2}\left(h^{\prime}\right)-C_{\mathrm{SL}}^{2}}} d h^{\prime},
$$

where $h_{k}$ is a height at which $r, r_{a}$, and $s$ are known and is not necessarily equal to $h_{0}$. This set of equations has been used by numerous investigators to estimate ray paths (e.g., Bauer et al. 1958; Blake 1968; Doviak and Zrnić 1993), although they have oftentimes been applied only to specific propagation environments (e.g., upward propagation only).

If reflection, which involves a reversal of propagation direction, or trapping, which is the situation where a ray remains at a certain height, does not occur, then solution of (A7)-(A9) is accomplished by solving (A7) or (A8) for $h$ given $C_{\mathrm{SL}}$ and $r$ or $r_{a}$ and using the resulting $h$ in (A9) to calculate $s$. Herein, the integrals in (A7)-(A9) were evaluated using Romberg integration (Press et al. 1992, section 4.3) and (A7)-(A8) were solved using the secant method. All computations were performed using double precision arithmetic.

If reflection or trapping does occur, then the heights at which these occur must be determined first, as at these heights Snell's law of refraction breaks down (Born and Wolf 1999, sections 1.5.4, 1.6.2) and, because (A7)-(A9) depend upon Snell's law of refraction, (A7)-(A9) break down as well. In fact, at these heights the quantity in the radicals of (A7)-(A9) equals zero. To determine these heights, Snell's law is rewritten in the form

$\cos [\theta(h)]-1=\frac{\left(a+h_{R_{-} \mathrm{MSL}}+h_{0}\right)}{\left(a+h_{R_{-} \mathrm{MSL}}+h\right)} \frac{n\left(h_{0}\right)}{n(h)} \cos \left[\theta\left(h_{0}\right)\right]-1$.

For a spherically stratified atmosphere, $\cos [\theta(h)]$ is restricted to be between 0 and 1 , and thus the lhs of (A10) is restricted to be between -1 and 0 . The value of the first term on the rhs of (A10), however, could exceed 1. This occurs at heights beyond a reflection height with, then, the rhs of (A10) equaling 0 at a reflection/trapping height and exceeding 0 at heights beyond a reflection height (and remaining at zero if the height in question is a trapping height). Reflection/trapping heights are identified by finding roots of the rhs of (A10), which herein is accomplished by 1) sampling the rhs of (A10) periodically through the range of heights of interest to bracket any potential roots, 2) obtaining estimated root values using the bisection method, and 3) refining estimated root values by taking small steps in height until the actual root is reached. A height is identified as a reflection or trapping height by evaluating the value of the first derivative of the rhs of (A10) at that height, with trapping occurring if this value is 0 and reflection occurring otherwise.

When reflection/trapping heights exist, (A7)-(A9) are used to determine radar data locations except in the immediate vicinity of reflection/trapping heights as use of (A7)-(A9) there would result in either numerical overflow near the reflection/trapping height or an error right at the reflection/trapping height. In the immediate vicinity of reflection/trapping heights, discretized versions of (A4)(A6) are used to determine $h$ and $s$ values through a stepwise process:

$$
\begin{aligned}
& \Delta h= \pm \frac{\sqrt{\left(a+h_{R_{-} \mathrm{MSL}}+h\right)^{2} n^{2}(h)-C_{\mathrm{SL}}^{2}}}{\left(a+h_{R_{-} \mathrm{MSL}}+h\right) n(h)} \Delta r, \\
& \Delta h= \pm \frac{\sqrt{\left(a+h_{R_{-} \mathrm{MSL}}+h\right)^{2} n^{2}(h)-C_{\mathrm{SL}}^{2}}}{\left(a+h_{R_{-} \mathrm{MSL}}+h\right) n^{2}(h)} \Delta r_{a},
\end{aligned}
$$

and

$$
\begin{aligned}
\Delta s & = \pm \frac{a C_{\mathrm{SL}}}{\left(a+h_{R_{-} \mathrm{MSL}}+h\right) \sqrt{\left(a+h_{R_{-} \mathrm{MSL}}+h\right)^{2} n^{2}(h)-C_{\mathrm{SL}}^{2}}} \Delta h \\
& =\frac{a C_{\mathrm{SL}}}{\left(a+h_{R_{-} \mathrm{MSL}}+h\right)^{2} n(h)} \Delta r \\
& =\frac{a C_{\mathrm{SL}}}{\left(a+h_{R_{-} \mathrm{MSL}}+h\right)^{2} n^{2}(h)} \Delta r_{a},
\end{aligned}
$$

where (A11) and (A12) are used in (A13) and the " \pm " symbol is not needed in the last two forms in (A13) since $s$ always increases with increasing $r$ and $r_{a}$. In this process care must be taken to ensure that one does not step beyond the reflection/trapping height. Once a reflection/trapping height is reached, a new value of $C_{\mathrm{SL}}$ at that height is computed for the next set of propagation calculations. Herein, the set of locations determined on the way to a reflection height are stored and used to step away from a reflection height to ensure that the solution is symmetric about the reflection height. Finally, it was found that (A7)-(A9) could generally be used to within $\sim 1 \mathrm{~cm}$ of a reflection/trapping height without numerical overflow occurring.

Another special circumstance arises when the initial elevation angle is $0^{\circ}$. In that situation, since $C_{\mathrm{SL}}=\left(a+h_{R \_\mathrm{MSL}}+h_{a}\right) n\left(h_{a}\right)$, the quantity in the radicals in (A7)-(A9) is zero at $h^{\prime}=h_{a}$. Thus (A11)-(A13) do not provide any assistance because they result in rays always being trapped at $h_{a}$, which generally is not the case. Blake (1968) dealt with this situation by replacing the quantity in the radicals in (A7)-(A9) with Taylor series approximations that do not have a value of zero at $h^{\prime}=h_{a}$. This, however, does not seem to be the best option since the quantity in these radicals truly should be zero since $d h / d r, d h / d r_{a}$, and $d h / d s$ are zero at this point [see (A4)-(A6)]. Consequently, a different approach was used. In this approach, the constant curvature model developed herein is applied to a very shallow layer from $h_{a}$ to $h_{a}+\Delta h_{\text {ipu }}$ (generally $\Delta h_{\text {ipu }}= \pm 1 \mathrm{~cm}$ was used, where the "ipu" stands for "initial point undefined"). To do this, the average ray curvature $\bar{C}$ in this layer is computed using $\bar{C}=\int_{h^{\prime}=h_{a}}^{h^{\prime}=h_{a}+\Delta h_{\text {ipu }}} C\left(h^{\prime}\right) d h^{\prime} / \int_{h^{\prime}=h_{a}}^{h^{\prime}} h_{a}+\Delta h_{\text {ipu }} d h^{\prime}$, where $C(h)$ is given by (6). If $\bar{C}=0$, the atmosphere is effectively homogeneous in this layer and $h$ and $s$ values for points in this layer are computed using (16) and (17). If $\bar{C} \neq 0$, then $h$ and $s$ values for points in this layer are computed using (13a) and [15a(1)]. It is noted that because in (13)-(23) $r$ is the actual, not apparent, $r$, one must compute $r$ values that correspond to $r_{a}$ values if one is seeking $h$ 
and $s$ values as a function of $r_{a}$. For this layer this is accomplished using $r_{a}=\bar{n} r$, where $\bar{n}=\int_{h^{\prime}=h_{a}}^{h^{\prime}=h_{a}+\Delta h_{\mathrm{ipu}}} n\left(h^{\prime}\right) d h^{\prime} / \int_{h^{\prime}=h_{a}}^{h^{\prime}} h_{a}+\Delta h_{\mathrm{ipu}} d h^{\prime}$.

\section{APPENDIX B}

\section{Derivation of Relation between Ray Tracing and SECC Model}

Differential equations for ray tracing are provided in appendix A. Here, with the assumption of constant curvature, a result that is consistent with the SECC model is produced through solution of (A4).

The starting point is expression of (A4) in terms of $r_{c}$ :

$$
\frac{d r}{d h}= \pm \frac{1}{\sqrt{1-\frac{n^{2}(h)}{r_{c}^{2}}\left(\frac{d h}{d n}\right)^{2}}}= \pm \frac{1}{\sqrt{1-\frac{1}{r_{c}^{2}}\left(\frac{d h}{d \ln n}\right)^{2}}}
$$

where (6) has been utilized. Assuming $r_{c}$ is constant, the solution to (6) is

$$
n(h)=\frac{2 r_{c} C_{\mathrm{SL}}}{h^{2}+2\left(a+h_{R_{-} \mathrm{MSL}}\right) h-h_{0}^{2}-2\left(a+h_{R_{-} \mathrm{MSL}}\right) h_{0}+\frac{2 r_{c} C_{\mathrm{SL}}}{n\left(h_{0}\right)}},
$$

where $h_{0}$ and $n\left(h_{0}\right)$ are the reference values (lower integral limits) used in the integrals that arise when deriving this relation. From (B2),

$$
\frac{d h}{d \ln n}=-\frac{h^{2}+2\left(a+h_{R_{-} \mathrm{MSL}}\right) h-h_{0}^{2}-2\left(a+h_{R_{-} \mathrm{MSL}}\right) h_{0}+\frac{2 r_{c} C_{S L}}{n\left(h_{0}\right)}}{2 h+2\left(a+h_{R_{-} \mathrm{MSL}}\right)},
$$

which, when substituted into (B1), produces

$$
\frac{d r}{d h}= \pm \frac{1}{\sqrt{1-\frac{1}{r_{c}^{2}}\left[\frac{h^{4}+c_{3} h^{3}+c_{2} h^{2}+c_{1} h+c_{0}}{4\left(a+h_{R_{-} \mathrm{MSL}}+h\right)^{2}}\right]}},
$$

where

$$
\begin{aligned}
c_{3} & =4\left(a+h_{R_{-} \mathrm{MSL}}\right) \\
c_{2} & =2\left[2 r_{c} \frac{C_{\mathrm{SL}}}{n\left(h_{0}\right)}+2\left(a+h_{R_{-} \mathrm{MSL}}\right)^{2}-2\left(a+h_{R_{-} \mathrm{MSL}}\right) h_{0}-h_{0}^{2}\right]=2\left[\sqrt{c_{0}}+2\left(a+h_{R_{-} \mathrm{MSL}}\right)^{2}\right] \\
c_{1} & =4\left(a+h_{R_{-} \mathrm{MSL}}\right)\left[2 r_{c} \frac{C_{\mathrm{SL}}}{n\left(h_{0}\right)}-2\left(a+h_{R_{-} \mathrm{MSL}}\right) h_{0}-h_{0}^{2}\right]=4\left(a+h_{R_{-} \mathrm{MSL}}\right) \sqrt{c_{0}} \\
c_{0} & =\left[2 r_{c} \frac{C_{\mathrm{SL}}}{n\left(h_{0}\right)}-2\left(a+h_{R_{-} \mathrm{MSL}}\right) h_{0}-h_{0}^{2}\right]^{2} \\
& =\left[2 r_{c}\left(a+h_{R_{-} \mathrm{MSL}}+h_{0}\right) \cos \left[\theta\left(h_{0}\right)\right]-2\left(a+h_{R_{-} \mathrm{MSL}}\right) h_{0}-h_{0}^{2}\right]^{2} .
\end{aligned}
$$

With the assumption that the lower limit altitude is $h_{0}=h_{a}$, the following approximations are applied:

$$
h^{4}+c_{3} h^{3}+c_{2} h^{2}+c_{1} h+c_{0} \approx c_{2}^{\prime} h^{2}+c_{1}^{\prime} h+c_{0}^{\prime},
$$

where

$$
\begin{aligned}
c_{2}^{\prime} & =4 r_{c}\left(a+h_{R_{-} \mathrm{MSL}}+h_{a}\right) \cos \theta \\
& =\overbrace{c_{1}}^{o\left(16 a^{2} \cos \theta\right)}-[\overbrace{4\left(a+h_{R_{-} \mathrm{MSL}}\right)^{2}}^{o\left(4 a^{2}\right)}-\overbrace{4\left(a+h_{R_{-} \mathrm{MSL}}\right) h_{a}}^{o\left(4 a^{2} / 300\right)}-\overbrace{2 h_{a}^{2}}^{o\left(2 a^{2} / 300^{2}\right)}] \\
c_{1}^{\prime} & =8 r_{c}\left(a+h_{R_{-} \mathrm{MSL}}\right)\left(a+h_{R_{-} \mathrm{MSL}}+h_{a}\right) \cos \theta \\
& =\overbrace{c_{1}}^{o\left(32 a^{3} \cos \theta\right)}+[\overbrace{8\left(a+h_{R_{-} \mathrm{MSL}}\right)^{2} h_{a}}^{o\left(8 a^{3} / 300\right)}+\overbrace{4\left(a+h_{R_{-} \mathrm{MSL}}\right) h_{a}^{2}}^{o\left(4 a^{3} / 300^{2}\right)}] \\
c_{0}^{\prime} & =\left[2 r_{c}\left(a+h_{R_{-} \mathrm{MSL}}+h_{a}\right) \cos \theta-2\left(a+h_{R_{-} \mathrm{MSL}}\right) h_{a}-h_{a}^{2}\right]^{2}-\left[2 h_{a}\left(a+h_{R_{-} \mathrm{MSL}}\right)+h_{a}^{2}\right]^{2} \\
& =\overbrace{c_{0}}^{o\left(4 a^{4} / 300^{2}\right)}-\overbrace{\left[2 h_{a}\left(a+h_{R_{-} \mathrm{MSL}}\right)+h_{a}^{2}\right]^{2}},
\end{aligned}
$$


TABLE B1. Characteristics of the polynomial terms in (B6). The second column provides the approximate scales of the polynomial terms using the largest term in the coefficients. The third column provides the ratio of a polynomial relative to the next polynomial term (e.g., term 0/term1). The final column provides the ratio of the leading terms in each coefficient relative to the next largest term in that coefficient.

\begin{tabular}{cccc}
\hline \hline Polynomial term & Term scale & Ratio to following term & Coefficient terms ratio \\
\hline 0 & $64 a^{4} \cos ^{2} \theta$ & $600 \cos \theta$ & $1440000 \cos ^{2} \theta$ \\
1 & $\frac{32}{300} a^{4} \cos \theta$ & 600 & $1200 \cos \theta$ \\
2 & $\frac{16}{300^{2}} a^{4} \cos \theta$ & $1200 \cos \theta$ & $4 \cos \theta$ \\
3 & $\frac{4}{300^{3}} a^{4}$ & 1200 & - \\
4 & $\frac{1}{300^{4}} a^{4}$ & - & - \\
\hline
\end{tabular}

scaling values are provided for different terms, and scaling parameters for typical propagation conditions and tropospheric applications are used with $h \simeq h_{a} \sim O(a / 300)$ and $r_{c} \sim$ $O(4 a)$. As shown in Table B1, the magnitudes of the terms in the quartic polynomial in (B6) significantly decrease with increasing degree. Moreover, the leading terms in the coefficients (involving $\cos \theta$ ) are generally much larger than the terms being added/subtracted to produce the approximation on the rhs of (B6). An exception occurs for very small values of $\cos \theta$, at which point nonrefractive conditions (infinite radius of curvature) are being approached.

Given (B6) and (B7), (B4) can be expressed as

$$
\frac{d r}{d h} \approx \pm \frac{\left(a+h_{R_{-} \mathrm{MSL}}+h\right)}{\sqrt{\left(a+h_{R_{-} \mathrm{MSL}}+h_{a}\right)^{2} \sin ^{2} \theta-\left[1-\frac{\left(a+h_{R_{-} \mathrm{MSL}}+h_{a}\right)}{r_{c}} \cos \theta\right]\left[\left(a+h_{R_{-} \mathrm{MSL}}+h_{a}\right)^{2}-\left(a+h_{R_{-} \mathrm{MSL}}+h\right)^{2}\right]}},
$$

which is the derivative with respect to $h$ of

$$
r \approx \frac{-c_{q 1} \pm \sqrt{c_{q 1}^{2}-4 c_{q 2} c_{q 0}}}{2 c_{q 2}}
$$

where

$$
\begin{aligned}
& c_{q 2}=\left[1-\frac{\left(a+h_{R_{-} \mathrm{MSL}}+h_{a}\right)}{r_{c}} \cos \theta\right] \\
& c_{q 1}=2\left(a+h_{R_{-} \mathrm{MSL}}+h_{a}\right) \sin \theta \\
& c_{q 0}=\left(a+h_{R_{-} \mathrm{MSL}}+h_{a}\right)^{2}-\left(a+h_{R_{-} \mathrm{MSL}}+h\right)^{2} .
\end{aligned}
$$

The relation (B9) is the solution of the quadratic

$$
\begin{aligned}
& {\left[1-\frac{\left(a+h_{R_{-} \mathrm{MSL}}+h_{a}\right)}{r_{c}} \cos \theta\right] r^{2}+2\left(a+h_{R_{-} \mathrm{MSL}}+h_{a}\right) \sin \theta r} \\
& +\left(a+h_{R_{-} \mathrm{MSL}}+h_{a}\right)^{2}-\left(a+h_{R_{-} \mathrm{MSL}}+h\right)^{2} \approx 0 .
\end{aligned}
$$

Expressing this quadratic in terms of $h$ produces

$$
h \approx\left[r^{2}+\left(a+h_{R_{-} \mathrm{MSL}}+h_{a}\right)^{2}+2 r\left(a+h_{R_{-} \mathrm{MSL}}+h_{a}\right)\left(\sin \theta-\frac{r}{2 r_{c}} \cos \theta\right)\right]^{1 / 2}-\left(a+h_{R_{-} \mathrm{MSL}}\right),
$$

which is $(13 c)$.

\section{REFERENCES}

Appleton, E., 1946: The influence of tropospheric conditions on ultra-short-wave propagation. Meteorological Factors in RadioWave Propagation, Physical Society and Royal Meteorological Society, $17 \mathrm{pp}$.

Bauer, J. R., W. C. Mason, and F. A. Wilson, 1958: Radio refraction in a cool exponential atmosphere, MIT Lincoln Laboratory Tech. Rep. 186, 29 pp.
Bean, B. R., and E. J. Dutton, 1966: Radio Meteorology. National Bureau of Standards Monogr., No. 92, U.S. Government Printing Office, 435 pp., https://doi.org/10.6028/ nbs.mono.92.

Bent, A. E., P. M. Austin, and M. L. Stone, 1950: Beam width and pulse length in radar weather detection, MIT Dept. of Meteorology Tech. Rep. 12, 61 pp.

Blake, L. V., 1968: Ray height computation for a continuous nonlinear atmospheric refractive-index profile. Radio Sci., 3, 8592, https://doi.org/10.1002/rds19683185. 
Born, M., and E. Wolf, 1999: Principles of Optics. 7th ed. Cambridge University Press, 986 pp.

Coleman, C. J., 2004: On the generalization of Snell's law. Radio Sci., 39, RS2005, https://doi.org/10.1029/2003RS003002.

Davies-Jones, R., V. T. Wood, and M. A. Askelson, 2019: Ray curvature on a flat Earth for computing virtual WSR-88D signatures of simulated supercell storms. Mon. Wea. Rev., 147, 1065-1075, https://doi.org/10.1175/MWR-D-18-0356.1.

Doviak, R. J., and D. S. Zrnić, 1993: Doppler Radar and Weather Observations. 2nd Ed. Academic Press, 562 pp.

Gao, J., K. Brewster, and M. Xue, 2006: A comparison of the radar ray path equations and approximations for use in radar data assimilation. Adv. Atmos. Sci., 23, 190-198, https://doi.org/ 10.1007/s00376-006-0190-3.

Gunashekar, S. D., E. M. Warrington, D. R. Siddle, and P. Valtr, 2007: Signal strength variations at $2 \mathrm{GHz}$ for three sea paths in the British Channel Islands: Detailed discussion and propagation modeling. Radio Sci., 42, RS4020, https://doi.org/ 10.1029/2006RS003617.

Harrington, R. F., 1993: Field Computation by Moment Methods. IEEE/OUP Series on Electromagnetic Wave Theory, IEEE Press, $240 \mathrm{pp}$.

Hartree, D. R., J. G. L. Michel, and P. Nicolson, 1946: Practical methods for the solution of the equations of tropospheric refraction. Meteorological Factors in Radio-Wave Propagation, Physical Society and Royal Meteorological Society, 127-168.

Johnson, R. S., 2010: Using electromagnetic ray tracing to develop a refractivity climatology for eastern North Dakota. M.S. thesis, Dept. of Atmospheric Sciences, University of North Dakota, 206 pp.

Kursinski, E. R., G. A. Hajj, S. S. Leroy, and B. Herman, 2000: The GPS radio occultation technique. Terr. Atmos. Ocean. Sci., 11, 53-114, https://doi.org/10.3319/TAO.2000.11.1.53(COSMIC).
Levis, C. A., J. T. Johnson, and F. L. Teixeira, 2010: Radiowave Propagation: Physics and Applications. John Wiley and Sons, 301 pp.

Levy, M., 2000: Parabolic Equation Methods for Electromagnetic Wave Propagation. Electromagnetic Wave Series, Vol. 45, Institution of Electrical Engineers, 347 pp.

Millington, G., 1957: The concept of the equivalent radius of the Earth in tropospheric propagation. Marconi Rev., 20, 79-93.

NIMA, 2000: Department of Defense World Geodetic System 1984. NIMA Tech. Rep. 8350.2, 3rd ed., 175 pp, https:// earth-info.nga.mil/GandG/publications/tr8350.2/wgs84fin.pdf.

Polyanin, A. D., and V. F. Zaitsev, 2003: Handbook of Exact Solutions for Ordinary Differential Equations. 2nd ed. Chapman and Hall/CRC, 787 pp.

Press, W. H., S. A. Teukolsky, W. T. Vetterling, and B. P. Flannery, 1992: Numerical Recipes in Fortran 77: The Art of Scientific Computing. 2nd ed. Vol. 1, Fortran Numerical Recipes, Cambridge University Press, 933 pp.

Schelleng, J. C., C. R. Burrows, and E. B. Ferrell, 1933: Ultra-shortwave propagation. Proc. Inst. Radio Engr., 21, 427-463, https:// doi.org/10.1109/JRPROC.1933.227639.

Stratton, J. A., 1941: Electromagnetic Theory. McGraw-Hill, $615 \mathrm{pp}$.

Taflove, A., and S. C. Hagness, 2005: Computational Electrodynamics: The Finite-Difference Time-Domain Method. 3rd ed. Artech House Publishers, 1038 pp.

Tseng, W.-K., and H.-S. Lee, 2010: Navigation on a great ellipse. J. Mar. Sci. Technol., 18, 369-375.

Wolfram Research, 2011: Mathematica Version 8.0.1.0. Wolfram Research.

Zeng, Y., U. Blahak, M. Neuper, and D. Jerger, 2014: Radar beam tracing methods based on atmospheric refractive index. J. Atmos. Oceanic Technol., 31, 2650-2670, https://doi.org/ 10.1175/JTECH-D-13-00152.1. 